\title{
Search for narrow resonances and quantum black holes in inclusive and b-tagged dijet mass spectra from pp collisions at $\sqrt{s}=7 \mathrm{TeV}$
}

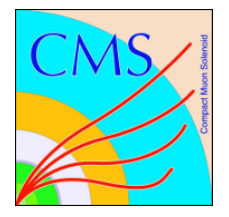

\section{The CMS collaboration}

E-mail: cms-publication-committee-chair@cern.ch

ABSTRACT: A search for narrow resonances and quantum black holes is performed in inclusive and b-tagged dijet mass spectra measured with the CMS detector at the LHC. The data set corresponds to $5 \mathrm{fb}^{-1}$ of integrated luminosity collected in pp collisions at $\sqrt{s}=7 \mathrm{TeV}$. No narrow resonances or quantum black holes are observed. Modelindependent upper limits at the $95 \%$ confidence level are obtained on the product of the cross section, branching fraction into dijets, and acceptance for three scenarios: decay into quark-quark, quark-gluon, and gluon-gluon pairs. Specific lower limits are set on the mass of string resonances $(4.31 \mathrm{TeV})$, excited quarks $(3.32 \mathrm{TeV})$, axigluons and colorons $(3.36 \mathrm{TeV})$, scalar color-octet resonances $(2.07 \mathrm{TeV}), \mathrm{E}_{6}$ diquarks $(3.75 \mathrm{TeV})$, and on the masses of $\mathrm{W}^{\prime}(1.92 \mathrm{TeV})$ and $\mathrm{Z}^{\prime}(1.47 \mathrm{TeV})$ bosons. The limits on the minimum mass of quantum black holes range from 4 to $5.3 \mathrm{TeV}$. In addition, b-quark tagging is applied to the two leading jets and upper limits are set on the production of narrow dijet resonances in a model-independent fashion as a function of the branching fraction to b-jet pairs.

KeYwORDS: Hadron-Hadron Scattering

ARXiv EPRINT: 1210.2387 


\section{Contents}

1 Introduction 1

2 The CMS detector and data sample 3

3 Event reconstruction and selection 4

4 Measurement of the dijet mass spectrum 5

5 Search for narrow dijet resonances and quantum black holes $\quad 6$

5.1 Statistical treatment and systematic uncertainties 9

$\begin{array}{llr}6 & \text { Results } & 10\end{array}$

$\begin{array}{llr}7 & \text { Summary } & 17\end{array}$

$\begin{array}{ll}\text { The CMS collaboration } & 23\end{array}$

\section{Introduction}

Events with two or more energetic jets in the final state are copiously produced in protonproton (pp) collisions at the Large Hadron Collider (LHC). Such events arise when the constituent partons are scattered with large transverse momenta $p_{\mathrm{T}}$. The invariant mass spectrum of the dijet system, consisting of the two jets with the largest $p_{\mathrm{T}}$ (leading jets), is predicted by quantum chromodynamics (QCD) to fall steeply and smoothly. However, there are numerous extensions of the standard model (SM) that predict the existence of new massive particles that couple to quarks (q) and gluons (g), and result in the appearance of resonant structures in the dijet mass spectrum. Furthermore, the dijet mass spectrum can be used to search for quantum black holes. Hence, dijet events provide one of the event topologies used to search for new physics.

In this Letter we report on a search for narrow resonances in the inclusive dijet mass spectrum measured with the Compact Muon Solenoid (CMS) detector at the LHC in pp collisions at a center-of-mass energy of $7 \mathrm{TeV}$. We complement the generic search with a more flavour-specific analysis, in which information based on displaced secondary vertices is used to identify jets resulting from the hadronization and decay of a b quark. As a consequence, the analysis has an enhanced sensitivity to objects that decay preferentially into $\mathrm{b} \bar{b}$ pairs.

Although the results of this search are applicable to any massive narrow resonance decaying to two jets, we consider specific models predicting the following narrow $s$-channel dijet resonances: 
- String resonances $(\mathrm{S})$, which are Regge excitations of quarks and gluons in string theory and decay predominantly to qg $[1,2]$.

- Scalar diquarks (D), which decay to qq and $\overline{\mathrm{q}} \overline{\mathrm{q}}$, predicted by a grand unified theory based on the $\mathrm{E}_{6}$ gauge symmetry group [3].

- Mass-degenerate excited quarks $\left(\mathrm{q}^{*}\right)$, which decay to $\mathrm{qg}$, predicted in quark compositeness models $[4,5]$; the compositeness scale is set to be equal to the mass of the excited quark.

- Axial-vector particles called axigluons (A), which decay to $\mathrm{q} \overline{\mathrm{q}}$, predicted in a model where the symmetry group $\mathrm{SU}(3)$ of QCD is replaced by the chiral symmetry $\mathrm{SU}(3)_{\mathrm{L}} \times$ $\mathrm{SU}(3)_{\mathrm{R}}[6]$.

- Color-octet colorons $(\mathrm{C})$, which also decay to $\mathrm{q} \overline{\mathrm{q}}$; these are vector particles predicted by the flavour-universal coloron model, in which the SU(3) gauge symmetry of QCD is embedded in a larger gauge group [7].

- Scalar color-octet resonances (S8) [8] that appear in many dynamical electroweak symmetry breaking models such as Technicolor. We consider the decay channel into a pair of gluons.

- Massive scalar color-octet resonances $\left(\mathrm{S} 8_{\mathrm{b}}\right)[9]$ that result from the breaking of an $\mathrm{SU}(3) \times \mathrm{SU}(3)$ gauge symmetry down to the QCD gauge group and that may have generically large couplings to $\mathrm{b}$ quarks. We consider the production of a coloron that subsequently decays into an $\mathrm{S} 8_{\mathrm{b}}$ and a light scalar singlet. We fix the singlet mass to $150 \mathrm{GeV}$. The $\mathrm{S} 8_{\mathrm{b}}$ and scalar singlet have branching fractions $(B)$ of approximately $100 \%$ to $\mathrm{b} \overline{\mathrm{b}}$ and $\mathrm{gg}$, respectively. The tangent of the mixing angle $\theta$ between the two $\mathrm{SU}(3)$ gauges is set to 0.15 . This resonance search is inclusive of extra jets, so the search strategy is insensitive to the decay of the low-mass singlet state.

- New gauge bosons ( $\mathrm{W}^{\prime}$ and $\left.\mathrm{Z}^{\prime}\right)$, that decay to $\mathrm{q} \overline{\mathrm{q}}$, predicted by models that include new gauge symmetries [10]; the $\mathrm{W}^{\prime}$ and $\mathrm{Z}^{\prime}$ bosons are assumed to have standardmodel-like couplings. Consequently, the ratio between the branching fraction of the $\mathrm{Z}^{\prime}$ to $\mathrm{b} \overline{\mathrm{b}}$ and the branching fraction to a pair of quarks (excluding the top quark) is approximately 0.22 .

- Randall-Sundrum (RS) gravitons $(\mathrm{G})$, which decay to $\mathrm{q} \overline{\mathrm{q}}$ and gg, predicted in the RS model of extra dimensions [11]. The value of the dimensionless coupling $k / \bar{M}_{\mathrm{Pl}}$ is chosen to be 0.1 , where $k$ is the curvature scale in the 5 -dimensional anti de Sitter space and $\bar{M}_{\mathrm{Pl}}$ is the reduced Planck scale. The ratio between the branching fraction of the RS graviton to $\mathrm{b} \overline{\mathrm{b}}$ and the branching fraction to a pair of quarks (excluding the top quark) or gluons is approximately 0.1 [12].

In addition, we report on a search for quantum black holes [13-15] in the inclusive dijet mass spectrum. This search is motivated by theories with low-scale quantum gravity, which 
offer a novel solution to the hierarchy problem of the standard model by lowering the scale of quantum gravity $M_{\mathrm{D}}$ from the Planck scale $\left(M_{\mathrm{Pl}} \sim 10^{16} \mathrm{TeV}\right)$ to a lower value $M_{\mathrm{D}} \sim 1 \mathrm{TeV}$, i.e. a value of the order of the electroweak symmetry breaking scale. Examples of models using this approach are the Arkani-Hamed-Dimopoulos-Dvali (ADD) model $[16,17]$ and the Randall-Sundrum (RS) model $[11,18]$. In the former model, extra dimensions are flat and compactified on a torus or a sphere, while in the latter model, a single extra dimension $(n=1)$ is warped. The strengthened gravity allows for formation of quantum black holes with masses $M_{\mathrm{QBH}}$ close to the quantum gravity scale $M_{\mathrm{D}}$. Such objects evaporate faster than they thermalize, resulting in a non-thermal decay into a pair of jets, rather than a highmultiplicity final state $[14,15]$. An earlier search for quantum black holes performed by the CMS experiment [19] was based on an analysis of high-multiplicity, energetic final states.

The searches presented in this document exceed the sensitivity to new physics of previous CMS [19-21] and ATLAS [22-24] published searches. A summary of recent searches for dijet resonances and a comparison of the approaches between different experiments are presented in ref. [25]. The most recent dedicated search for $b \bar{b}$ resonances in the dijet final state at a hadron collider was performed by the CDF experiment in Run I of the Tevatron [26].

\section{The CMS detector and data sample}

The CMS experiment uses a right-handed coordinate system, with the origin at the center of the detector. The $z$-axis points along the direction of the counterclockwise beam and the $\mathrm{x}$-axis points to the centre of the LHC; $\phi$ is the azimuthal angle, covering $-\pi<\phi \leq \pi$, $\theta$ is the polar angle, and the pseudorapidity $\eta \equiv-\ln [\tan (\theta / 2)]$.

The central feature of the CMS apparatus is a superconducting solenoid of $6 \mathrm{~m}$ internal diameter providing an axial magnetic field of $3.8 \mathrm{~T}$. Within the field volume in the central pseudorapidity region are the silicon-pixel and silicon-strip tracker $(|\eta|<2.4)$ and the barrel and endcap calorimeters $(|\eta|<3)$ consisting of a lead-tungstate crystal electromagnetic calorimeter (ECAL) and a brass/scintillator hadron calorimeter (HCAL). An iron/quartzfibre Cherenkov calorimeter is located in the forward region $(3<|\eta|<5)$, outside the field volume. For triggering purposes and to facilitate jet reconstruction, the ECAL and HCAL cells are grouped into towers projecting radially outward from the center of the detector. The energy deposits measured in the ECAL and the HCAL within each projective tower are summed to obtain the calorimeter tower energy. A more detailed description of the CMS detector, including its muon subdetectors, can be found elsewhere [27].

The integrated luminosity of the data sample used for this analysis is $4.98 \pm$ $0.11 \mathrm{fb}^{-1}[28]$, and corresponds to the full data sample recorded by the CMS experiment in 2011. Events are recorded using a two-tier trigger system. The sample was collected using a combination of triggers requiring the presence of jets in the event. At the start of the data-taking period, a multijet trigger based on $H_{\mathrm{T}}$ was used, where $H_{\mathrm{T}}$ is the scalar sum of the transverse momenta of all jets in the event with $p_{\mathrm{T}}$ above $40 \mathrm{GeV}$. Over the course of the data-taking period, the $H_{\mathrm{T}}$ threshold of the lowest unprescaled $H_{\mathrm{T}}$ trigger was increased from 350 to $750 \mathrm{GeV}$ to keep the overall trigger rate approximately constant 
as the number of additional pp collisions in the same or adjacent bunch crossings (pileup interactions) was increasing. To mitigate the negative impact of increasing $H_{\mathrm{T}}$ thresholds on the overall trigger efficiency, a dedicated dijet-mass trigger based on "wide-jet" reconstruction, the offline reconstruction technique described in section 3, was introduced toward the end of the data-taking period. Events with dijet masses greater than $850 \mathrm{GeV}$ and pseudorapidity separation between the two jets $|\Delta \eta|<2$ are selected online with this dedicated trigger. The efficiency of all of the triggers used in this analysis is measured from the data to be larger than $99.8 \%$ for dijet masses above $890 \mathrm{GeV}$.

\section{Event reconstruction and selection}

Events selected by the trigger system are required to be consistent with coming from a pp collision and have at least one reconstructed primary vertex within $\pm 24 \mathrm{~cm}$ of the detector center along the beam line and within $2 \mathrm{~cm}$ of the detector center in the plane transverse to the beam.

Jets are reconstructed offline using the anti- $k_{\mathrm{T}}$ clustering algorithm [29] with a distance parameter of 0.5. The four-momenta of particles reconstructed by the CMS particle-flow (PF) algorithm [30,31] are used as input to the jet-clustering algorithm. The particle-flow algorithm combines information from all CMS subdetectors to provide a complete list of long-lived particles in the event. Reconstructed and identified particles include muons, electrons (with associated bremsstrahlung photons), photons (including conversions in the tracker volume), and charged and neutral hadrons. The reconstructed jet energy $E$ is defined as the scalar sum of the energies of the constituents of the jet, and the jet momentum $\vec{p}$ as the vector sum of their momenta. The jet transverse momentum $p_{\mathrm{T}}$ is the component of $\vec{p}$ perpendicular to the beam. All reconstructed jets used in this analysis are required to pass identification criteria that are fully efficient for signal events [32], in order to remove possible instrumental and non-collision backgrounds in the selected sample. The mising transverse energy $E_{\mathrm{T}}^{\mathrm{miss}}$ is defined as the magnitude of the vector sum of the transverse momenta of all particles reconstructed in the event.

The jet energy scale is calibrated using jet energy corrections derived from Monte Carlo simulation, test beam results, and collision data [33]. The corrections account for extra energy clustered into jets from pileup interactions on an event-by-event basis [34]. Additional corrections for the flavor of the jet are small $(<1 \%)$ and are not applied; however, when b tagging is applied, the systematic uncertainty in the jet energy scale is increased to account for the different fragmentation and decay properties of heavy-flavor-originated jets.

Calibrated PF jets are clustered into what are called "wide jets" [21]. The wide jet reconstruction technique, inspired by performance studies of different jet definitions [35], increases the search sensitivity by recombining large-angle final-state QCD radiation from the outgoing partons, resulting in an improved dijet mass resolution. The clustering starts with the two leading jets, which are both required to have $|\eta|<2.5$. No explicit requirement on $p_{\mathrm{T}}$ of the two leading jets is applied. All other jets with $p_{\mathrm{T}}>30 \mathrm{GeV}$ and $|\eta|<2.5$ are added to the closest leading jet if they are within $\Delta R \equiv \sqrt{(\Delta \eta)^{2}+(\Delta \phi)^{2}}<1.1$, where $\Delta \eta$ and $\Delta \phi$ are the distances between the two jets in $\eta$ and $\phi$, respectively. In this way two 
wide jets are formed. Compared to our previous search [21], the minimum $p_{\mathrm{T}}$ threshold for subleading jets used in the wide-jet technique has been increased from $10 \mathrm{GeV}$ to $30 \mathrm{GeV}$ in order to be more robust against jets coming from pileup interactions.

The dijet system is composed of the two wide jets. We require that the pseudorapidity separation $\Delta \eta$ of the two wide jets satisfies $|\Delta \eta|<1.3$, and that both wide jets be in the region $|\eta|<2.5$. These requirements maximize the search sensitivity for isotropic decays of dijet resonances in the presence of QCD background. The dijet mass is given by $m=\sqrt{\left(E_{1}+E_{2}\right)^{2}-\left(\vec{p}_{1}+\vec{p}_{2}\right)^{2}}$, where $E_{1}\left(E_{2}\right)$ and $\vec{p}_{1}\left(\vec{p}_{2}\right)$ are the energy and momentum of the leading (next-to-leading) jet. For the trigger selection to be fully efficient, we select events with $m>890 \mathrm{GeV}$ without any requirement on wide-jet $p_{\mathrm{T}}$. To study possible impact of pileup on the analysis, the rate of selected events, defined as the number of events passing the event selection per unit of integrated luminosity, over the course of the data-taking period was analyzed. Despite the increasing pileup, the rate of selected events was found to be stable.

Jets from the hadronization and decay of b quarks are identified ("tagged") by the characteristically long lifetime of B hadrons. The combined-secondary-vertex (CSV) algorithm [36] uses variables from reconstructed secondary vertices together with track-based lifetime information to distinguish jets that originate from a b quark from those that originate from lighter quarks and gluons. This algorithm was tuned for $b$ jets from top-quark decays but shows good performance in other types of events as well. Based on a study of the expected upper limits and the properties of the tagger, the loose operating point of the CSV tagger was chosen for this analysis. The ratio of the tagging efficiency between data and simulation is measured in a b-quark-enriched sample [36]. This data-to-simulation "scale factor" is found to depend on the jet $p_{\mathrm{T}}$, but it is close to unity (within $\sim 5 \%$ ). A similar scale factor is measured for light jets passing the b-tagging criteria ("mistags") and is found to depend on the jet $p_{\mathrm{T}}$ and $\eta$, but it is also close to unity (within $\sim 10 \%$ ). Because of the limited number of jets at high $p_{\mathrm{T}}$, the scale factors are measured up to a jet $p_{\mathrm{T}}$ of $670 \mathrm{GeV}$ and are extrapolated to higher values of the jet $p_{\mathrm{T}}$. To take into account additional uncertainty associated with the extrapolation procedure, larger uncertainties are assigned to the extrapolated values of the scale factors. Only the leading subjet in each of the two wide jets is considered for $\mathrm{b}$ tagging. Therefore, events can be separated into three exclusive categories: 0,1 , and $2 \mathrm{~b}$ tags.

\section{Measurement of the dijet mass spectrum}

The dijet mass spectrum used to search for narrow dijet resonances is defined as

$$
\frac{\mathrm{d} \sigma}{\mathrm{d} m} \simeq \frac{1}{\int L \mathrm{~d} t} \frac{N_{i}}{\Delta m_{i}}
$$

where $m$ is the dijet mass, $N_{i}$ is the number of events in the $i$-th dijet mass bin, $\Delta m_{i}$ is the width of the $i$-th dijet mass bin, and $\int L \mathrm{~d} t$ is the integrated luminosity of the data sample. The size of dijet mass bins is approximately equal to the dijet mass resolution [20]. To test the smoothness of the measured dijet mass spectrum, we fit the following parameterization 
to the data:

$$
\frac{\mathrm{d} \sigma}{\mathrm{d} m}=\frac{P_{0}(1-m / \sqrt{s})^{P_{1}}}{(m / \sqrt{s})^{P_{2}+P_{3} \ln (m / \sqrt{s})}}
$$

where $P_{0}, P_{1}, P_{2}$, and $P_{3}$ are free parameters and $\sqrt{s}=7 \mathrm{TeV}$. This functional form has been used in previous searches $[20,22,23,37]$ to describe both data and QCD predictions.

Figure 1 (a) presents an inclusive dijet mass spectrum for the two wide jets, a fit to the data, and bin-by-bin fit residuals, defined as the difference between the data and the fit value divided by the statistical uncertainty in the data. The vertical error bars are central intervals with correct coverage for Poisson variation, and the horizontal error bars are the bin widths. The data are compared to a QCD prediction from PYTHIA [38] (v6.4.24), which includes a simulation of the CMS detector based on GEant4 [39, 40] (v4.9.4) and the jet energy corrections. The prediction uses a renormalization scale $\mu=p_{\mathrm{T}}$ of the hardscattered partons with the CTEQ6L1 parton distribution functions (PDFs) [41] and the $\mathrm{Z} 2$ underlying event tune (the Z2 tune is identical to the Z1 tune [42] except that Z2 uses the CTEQ6L1 PDFs), and has been normalized to the data by multiplying the prediction by a factor of 1.22 . This factor was derived by scaling the number of predicted events with $m>890 \mathrm{GeV}$ to that observed in data. The shape of the leading-order (LO) QCD prediction is in agreement with the data. Figures 1 (b), (c), and (d) present the dijet mass spectra, fits to the data, and the bin-by-bin fit residuals for the three b-tag multiplicity categories: 0,1 , and $2 \mathrm{~b}$ tags.

Based on the fit residuals and the values of the reduced $\chi^{2}$ obtained, no significant deviations from the fit function are observed in the measured dijet mass spectra, indicating that the data are well described by a smooth function.

\section{Search for narrow dijet resonances and quantum black holes}

We search for narrow dijet resonances, for which the natural resonance width is small compared to the CMS dijet mass resolution [25], and for quantum black holes. The dijet mass shape of narrow dijet resonances depends primarily on the type of partons coming from the resonance decay, because this affects both the amount of radiation and the response of the detector to final state jets. Using PYTHIA and the CMS detector simulation, the dijet mass shapes for the following parton pairings are predicted: $\mathrm{q} \overline{\mathrm{q}}$ (or qq) resonances from the process $G \rightarrow q \bar{q}[11], b \bar{b}$ resonances from $G \rightarrow b \bar{b}$ [11], qg resonances from $q^{*} \rightarrow q g$ [4], and $\mathrm{gg}$ resonances from $\mathrm{G} \rightarrow \mathrm{gg}$ [11]. The predicted dijet mass shapes have a Gaussian core coming from the jet energy resolution, and a tail towards lower mass arising from QCD radiation and steeply falling parton distribution functions. The dijet mass shapes are relatively narrow for $\mathrm{q} \overline{\mathrm{q}}$ (qq) resonances, wider for $\mathrm{b} \overline{\mathrm{b}}$ and $\mathrm{qg}$ resonances, and are the widest for gg resonances. The increase of the width of the measured mass shape and the shift of the mass distribution towards lower masses are enhanced when the number of gluons in the final state is larger, because gluons are more likely to radiate than quarks. The dijet mass shapes are wider for $b \bar{b}$ resonances because of the presence of neutrinos from the semileptonic b decays that escape detection. 


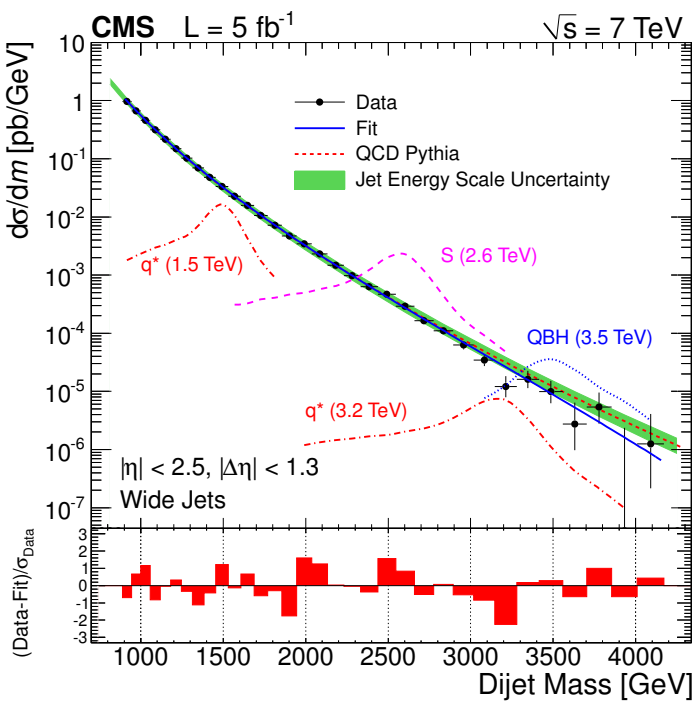

(a)

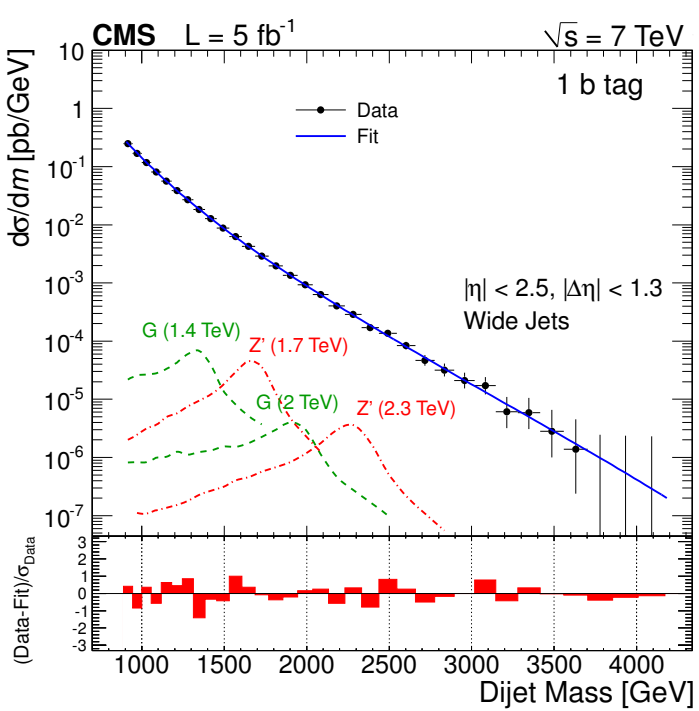

(c)

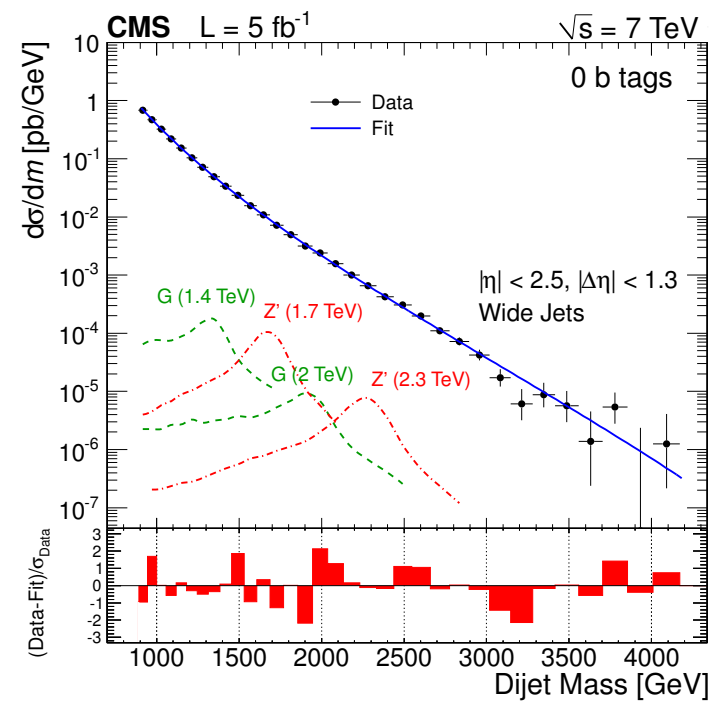

(b)

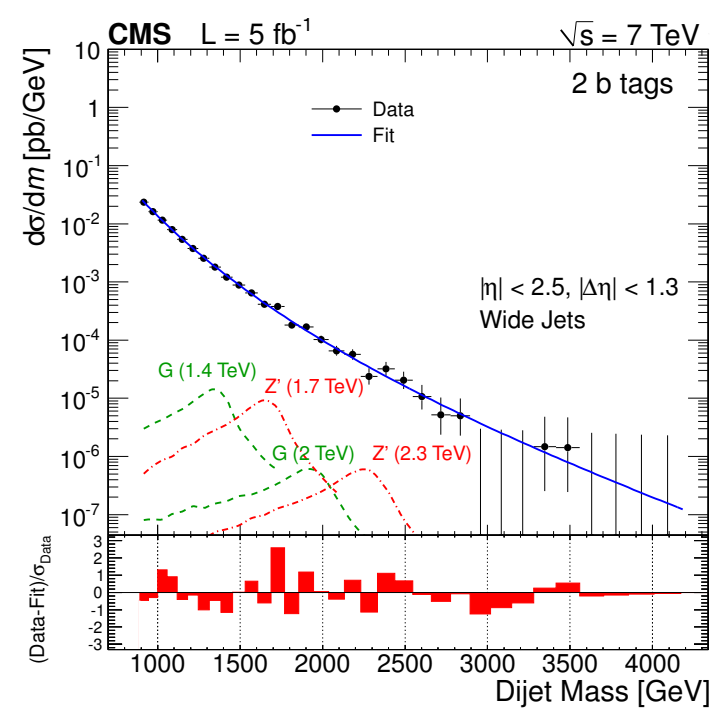

(d)

Figure 1. (a) Inclusive dijet mass spectrum from wide jets (points) compared to a smooth fit (solid) and predictions for QCD (short-dashed), excited quarks $\left(\mathrm{q}^{*}\right)$, string resonances (S), and quantum black holes (QBH). The QCD prediction has been normalized to the data (see text). The shaded band shows the contribution from the systematic uncertainty in the jet energy scale. (b), (c), (d) Dijet mass spectra from wide jets (points) for different b-tag multiplicity categories compared to a smooth fit (solid) and predictions for RS graviton $(\mathrm{G})$ and $\mathrm{Z}^{\prime}$. The bin-by-bin fit residuals are shown at the bottom of each plot. The functional form of the fit is described in the text.

It is commonly assumed $[43,44]$ that the minimum mass of quantum black holes $M_{\mathrm{QBH}}^{\min }$ cannot be smaller than $M_{\mathrm{D}}$. However, the formation threshold can be significantly larger than $M_{\mathrm{D}}$. For a given $M_{\mathrm{D}}$, the dijet mass shapes for quantum black holes are fairly independent of the number of extra dimensions $n$ and would appear as bumps in a steeply 


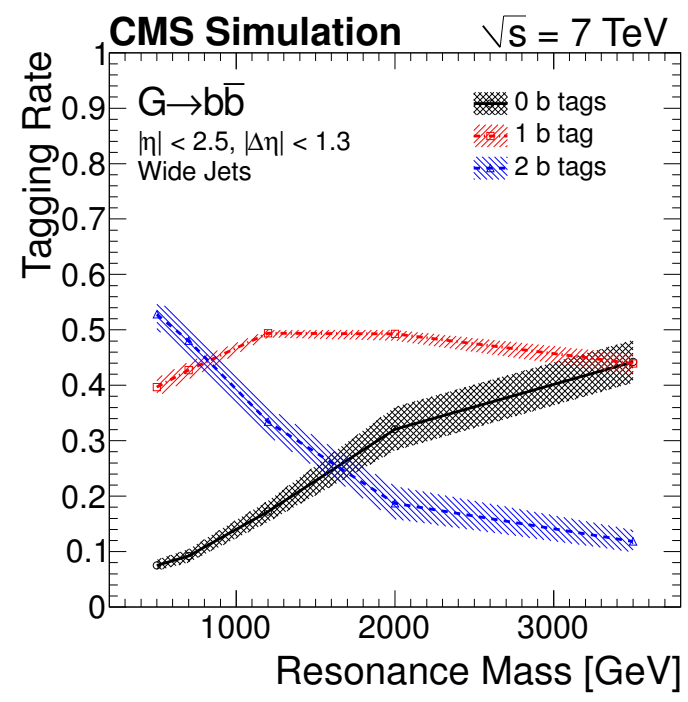

(a)

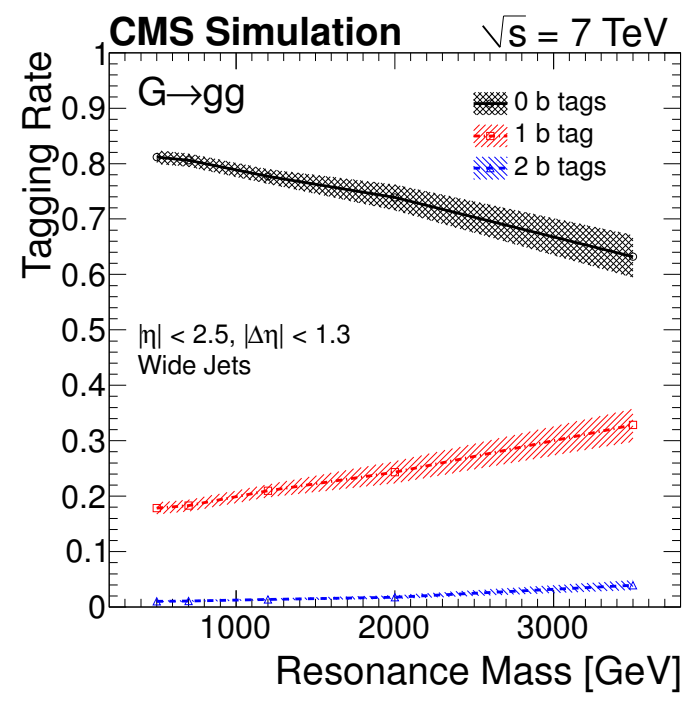

(b)

Figure 2. Tagging rates for 0,1 , and $2 \mathrm{~b}$ tags as a function of the resonance mass for (a) $b \bar{b}$ and (b) gg decay modes of the RS graviton $(\mathrm{G})$. Hatched regions represent uncertainties in the rates due to variations of the b-tag scale factors within their uncertainties. The tagging rates for the $q \bar{q}$ $(\mathrm{q}=\mathrm{u}, \mathrm{d}, \mathrm{s})$ decay modes are similar to the gg tagging rates.

falling QCD dijet mass spectrum, as shown in figure 1 (a). The dijet mass shapes for quantum black holes are modeled using the QBH (v1.03) matrix-element generator [45] with the CTEQ6L PDF set [41], followed by the parton showering simulation with PYTHIA and a fast parametric simulation of the CMS detector [46].

Based on the number of b-tagged jets, events are separated into three exclusive categories: 0-, 1-, and 2-tag categories. The tagging rate for each of these categories is defined as the fraction of events ending up in that category. The tagging rates as a function of the resonance mass are derived for different decay modes of RS gravitons and are shown in figure 2 for the $b \bar{b}$ and gg decay modes. As can be seen in the figure, the efficiency to correctly tag a $b$ jet decreases as the resonance mass increases. The rate of double-tagging a resonance that decays into two light quarks or gluons remains below $\sim 5 \%$ throughout the mass range. The tagging rates for the $\mathrm{q} \overline{\mathrm{q}}(\mathrm{q}=\mathrm{u}, \mathrm{d}, \mathrm{s})$ decay modes are similar to the gg tagging rates. The rate of double-tagging a resonance that decays into two charm quarks is systematically higher than for light flavor decay modes but is still significantly lower than for the $\mathrm{b} \overline{\mathrm{b}}$ decay mode (by a factor of $\sim 4$ at a resonance mass of $1 \mathrm{TeV}$ ). Rather than introduce an additional dependence of the result on the branching fraction to c quarks, we assume that the cic decay mode has the same tagging rates as the light quark and gluon decay modes. This assumption simplifies the interpretation of the analysis by removing an extra parameter at the cost of slightly reduced sensitivity. The tagging rates shown in figure 2 are assumed to be universally applicable to all narrow resonances decaying into the same type of partons. 


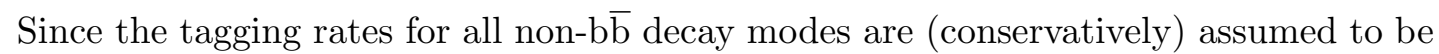
the same, the only free parameter that specifies the fraction of 0,1 , and 2 b-tag events originating from a narrow resonance with a given mass is the $\mathrm{b} \overline{\mathrm{b}}$ decay fraction $f_{\mathrm{b} \overline{\mathrm{b}}}$ defined at the parton level as

$$
f_{\mathrm{b} \overline{\mathrm{b}}}=\frac{B(\mathrm{X} \rightarrow \mathrm{b} \overline{\mathrm{b}})}{B(\mathrm{X} \rightarrow \mathrm{jj})}
$$

where $\mathrm{X}$ is a generic narrow resonance. As $f_{\mathrm{b} \overline{\mathrm{b}}}$ increases, the fraction of events from a resonance populating the $2 \mathrm{~b}$-tag spectrum is expected to increase, depending on the tagging rates shown in figure 2 . Because of the inefficiency in b tagging, even in the case of $f_{\mathrm{b} \overline{\mathrm{b}}}=1$, a fraction of events still populates the 0 and $1 \mathrm{~b}$-tag spectra.

\subsection{Statistical treatment and systematic uncertainties}

No significant deviations from the expected background have been observed in the measured dijet mass spectra. We use the measured dijet mass spectra, the background parameterization, and the dijet mass shapes to set upper limits on $\sigma \times B \times A$, the product of the production cross section $(\sigma)$, branching fraction $(B)$ for the jet-jet final state, and acceptance $(A)$ for the kinematic requirements $|\eta|<2.5$ and $|\Delta \eta|<1.3$. The acceptance for isotropic decays is $A \approx 0.6$, independent of the heavy resonance mass.

For setting upper limits, we use a Bayesian formalism [47] with a flat prior on the signal cross section, consistent with other dijet resonance searches at the LHC [21, 24]; log-normal priors are used to model systematic uncertainties, which are marginalized as nuisance parameters. We calculate the posterior probability density as a function of resonance cross section independently at each value of the resonance mass. With b tagging applied, the data from each of the three tagged spectra are combined into a single likelihood to provide a single limit by assuming a particular value for $f_{\mathrm{b} \overline{\mathrm{b}}}$.

In order to achieve good coverage properties for the confidence intervals in the presence of a signal that is not yet strong enough to be observed, the data are fit to the background function plus a signal line shape with the signal cross section treated as a free parameter. The resulting fit function with the signal cross section set to zero is used as the background hypothesis. The uncertainty in the background fit is incorporated by marginalizing over the background fit parameters (not including the signal cross section) after diagonalizing the covariance matrix to account for the correlations in the parameters. We also calculate the expected upper limits on $\sigma \times B \times A$ using pseudo-experiments: ensembles of simulated experiments generated from the smooth background parameterization obtained from the signal-plus-background fit to the data.

While events from a resonance that are double-tagged are dominated by the $b \bar{b}$ final state (assuming that $f_{\mathrm{b} \overline{\mathrm{b}}}$ is not trivially small), there remains an ambiguity for the 0 and 1 b-tag cases. Resonances such as the RS graviton decay into pairs of gluons as well as q $\bar{q}$ pairs. On the other hand, particles such as the $\mathrm{Z}^{\prime}$ or $\mathrm{S} 8_{\mathrm{b}}$ decay exclusively into $\mathrm{q} \overline{\mathrm{q}}$ final states. Because of the gluon's larger color factor, gluons radiate more than quarks, resulting in a broader dijet mass shape and, consequently, weaker expected limits. While the widejet reconstruction technique mitigates this effect, the limits depend on whether the 0 and $1 \mathrm{~b}$-tag mass shapes are dominated by gluons or quarks in the final state. Therefore, when 
$\mathrm{b}$ tagging is applied, two sets of upper limits are placed on $\sigma \times B \times A$, one for resonances that decay into gluons in addition to b quarks ("gg/bb") and one for resonances that decay into quarks only ("qq/bb"). Mass shapes appropriate to gg or qq resonances are used in conjuction with a bb mass shape used for both types of resonances. The mass shapes in each tag category are weighted according to the expected gluon, quark, or b-quark content, as determined by the tagging rates and $f_{\mathrm{b} \overline{\mathrm{b}}}$.

In the inclusive analysis, the dominant sources of systematic uncertainty are the jet energy scale $(2.2 \%)$, the jet energy resolution (10\%), the integrated luminosity determination $(2.2 \%)$ [28], and the statistical uncertainty in the background parameterization, where the uncertainties in the sources are given in parentheses. The statistical uncertainty in the background parameterization leads to the uncertainty in the expected background yields, with the double-tagged dijet mass spectrum having the largest uncertainty that ranges from $\sim 1 \%$ at a dijet mass of $1 \mathrm{TeV}$ to $\sim 15 \%$ at $3.5 \mathrm{TeV}$. The jet energy scale and the resolution uncertainties are incorporated into the limit-setting calculation by marginalizing over nuisance parameters that control the mean and the width of the dijet mass shape. For the b-tagged analysis, the uncertainties in the b-tag scale factors ( $\sim 5 \%$ for heavy and $\sim 10 \%$ for light flavor jets) [36] are also considered. The flavor dependence of the energy response for $\mathrm{PF}$ jets at high jet $p_{\mathrm{T}}(>100 \mathrm{GeV})$ relevant for this analysis is well within the jet energy scale uncertainty [33]; nevertheless, for the b-tagged analysis, the jet energy scale uncertainty is conservatively assigned to be $3 \%$ for all resonance masses considered.

\section{Results}

Figure 3 shows the observed upper limits at the $95 \%$ confidence level (CL) on $\sigma \times B \times A$ for qq, qg, and gg resonances from the inclusive analysis. The observed upper limits for signal masses between 1.0 and $4.3 \mathrm{TeV}$ are also reported in table 1 . The observed upper limits can be compared to predictions of $\sigma \times B \times A$ at the parton level, without any detector simulation, in order to determine mass limits on new particles. The theoretical predictions are obtained at LO with narrow width approximation using CTEQ6L1 parton distribution functions [41]. For $\mathrm{S} 8_{\mathrm{b}}$ resonances, a $\mathrm{LO}$ cross section is obtained using the MADGRAPH matrix-element generator [48]. For axigluons and colorons, we also take into account the next-to-leadingorder $K$-factors [49]. New particles are excluded at the 95\% CL in mass regions for which the theory curve lies above the upper limit for the appropriate pair of partons.

Figure 4 shows the expected limits and their uncertainty bands for qq, qg, and gg resonances and compares them to both the observed limits and theoretical predictions for new resonances. Upward fluctuations in data observed around 2 and $2.5 \mathrm{TeV}$ result in observed limits that are less stringent than the expected ones; conversely, a downward fluctuation around $3.2 \mathrm{TeV}$ results in more stringent observed limits than the expected ones.

For string resonances, we exclude masses smaller than $4.31 \mathrm{TeV}$; this extends our previous exclusion of $0.5<M(\mathrm{~S})<4.0 \mathrm{TeV}$ [20, 21]. For excited quarks, we exclude masses smaller than $3.32 \mathrm{TeV}$; this extends our previous exclusion of $0.5<M\left(\mathrm{q}^{*}\right)<$ $2.49 \mathrm{TeV}[20,21]$ and extends the ATLAS exclusion at $2.99 \mathrm{TeV}$ [24]. For $\mathrm{E}_{6}$ diquarks, we exclude masses in the range $1.0<M\left(\mathrm{E}_{6}\right)<3.75 \mathrm{TeV}$; this extends our previous exclu- 


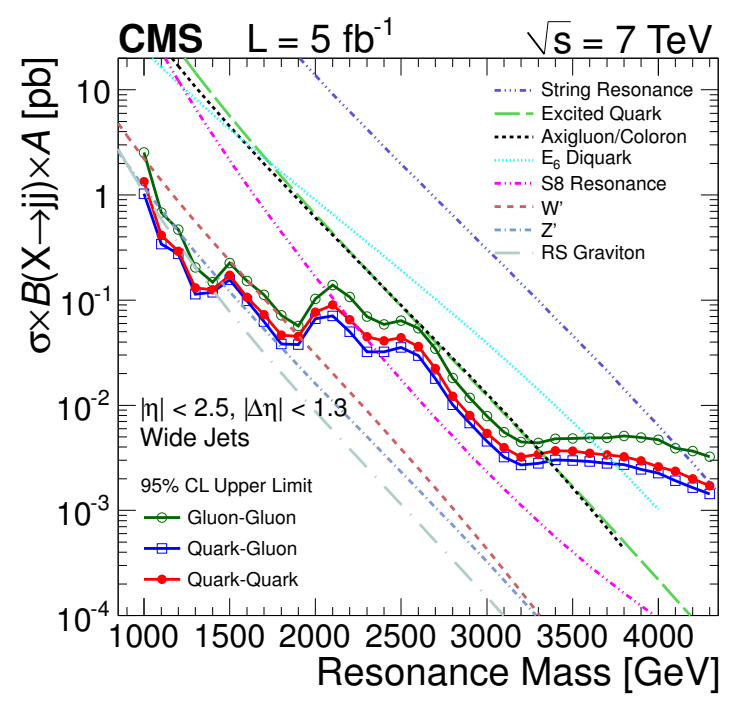

Figure 3. Observed 95\% CL upper limits on $\sigma \times B \times A$ for dijet resonances of type gluongluon (open circles), quark-gluon (solid circles), and quark-quark (open boxes) from the inclusive analysis, compared to theoretical predictions for string resonances $[1,2]$, excited quarks $[4,5]$, axigluons [6, 49], colorons [7], $\mathrm{E}_{6}$ diquarks [3], $\mathrm{S} 8$ resonances [8], $\mathrm{W}^{\prime}$ and $\mathrm{Z}^{\prime}$ bosons [10], and Randall-Sundrum gravitons [11].

\begin{tabular}{|c|c|c|c||c|c|c|c|}
\hline Mass & \multicolumn{3}{|c||}{ Upper limit on $\sigma \times B \times A[\mathrm{pb}]$} & \multicolumn{3}{|c|}{ Mass } & \multicolumn{3}{|c|}{ Upper limit on $\sigma \times B \times A[\mathrm{pb}]$} \\
{$[\mathrm{TeV}]$} & $\mathrm{qq}$ & $\mathrm{qg}$ & $\mathrm{gg}$ & {$[\mathrm{TeV}]$} & $\mathrm{qq}$ & $\mathrm{qg}$ & $\mathrm{gg}$ \\
\hline 1.0 & 1.0 & 1.3 & 2.5 & 2.7 & 0.018 & 0.022 & 0.035 \\
1.1 & 0.34 & 0.42 & 0.68 & 2.8 & 0.010 & 0.012 & 0.018 \\
1.2 & 0.28 & 0.29 & 0.47 & 2.9 & 0.0068 & 0.0080 & 0.0118 \\
1.3 & 0.11 & 0.13 & 0.20 & 3.0 & 0.0045 & 0.0054 & 0.0079 \\
1.4 & 0.12 & 0.13 & 0.15 & 3.1 & 0.0032 & 0.0039 & 0.0056 \\
1.5 & 0.16 & 0.17 & 0.23 & 3.2 & 0.0027 & 0.0032 & 0.0045 \\
1.6 & 0.10 & 0.11 & 0.15 & 3.3 & 0.0028 & 0.0034 & 0.0044 \\
1.7 & 0.062 & 0.073 & 0.112 & 3.4 & 0.0030 & 0.0037 & 0.0048 \\
1.8 & 0.038 & 0.046 & 0.072 & 3.5 & 0.0030 & 0.0037 & 0.0048 \\
1.9 & 0.038 & 0.045 & 0.057 & 3.6 & 0.0029 & 0.0035 & 0.0049 \\
2.0 & 0.066 & 0.077 & 0.103 & 3.7 & 0.0028 & 0.0034 & 0.0049 \\
2.1 & 0.071 & 0.090 & 0.139 & 3.8 & 0.0027 & 0.0032 & 0.0051 \\
2.2 & 0.050 & 0.065 & 0.107 & 3.9 & 0.0025 & 0.0030 & 0.0049 \\
2.3 & 0.032 & 0.045 & 0.070 & 4.0 & 0.0023 & 0.0026 & 0.0047 \\
2.4 & 0.032 & 0.041 & 0.059 & 4.1 & 0.0019 & 0.0024 & 0.0039 \\
2.5 & 0.035 & 0.044 & 0.064 & 4.2 & 0.0016 & 0.0020 & 0.0037 \\
2.6 & 0.030 & 0.036 & 0.054 & 4.3 & 0.0014 & 0.0017 & 0.0032 \\
\hline
\end{tabular}

Table 1. Observed 95\% CL upper limits on $\sigma \times B \times A$ for narrow quark-quark (qq), quarkgluon (qg) and gluon-gluon (gg) resonances with masses between 1.0 and $4.3 \mathrm{TeV}$, derived from an inclusive analysis of dijet mass spectra. 


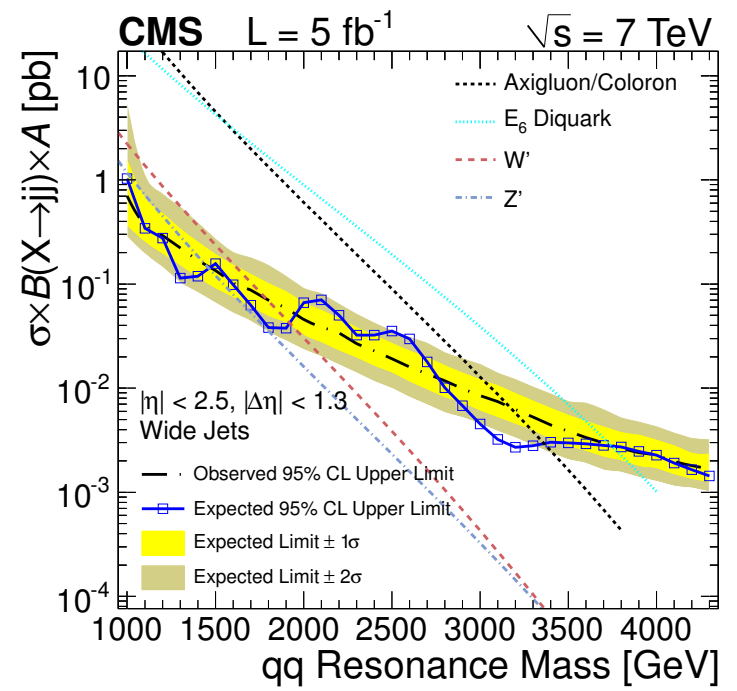

(a)

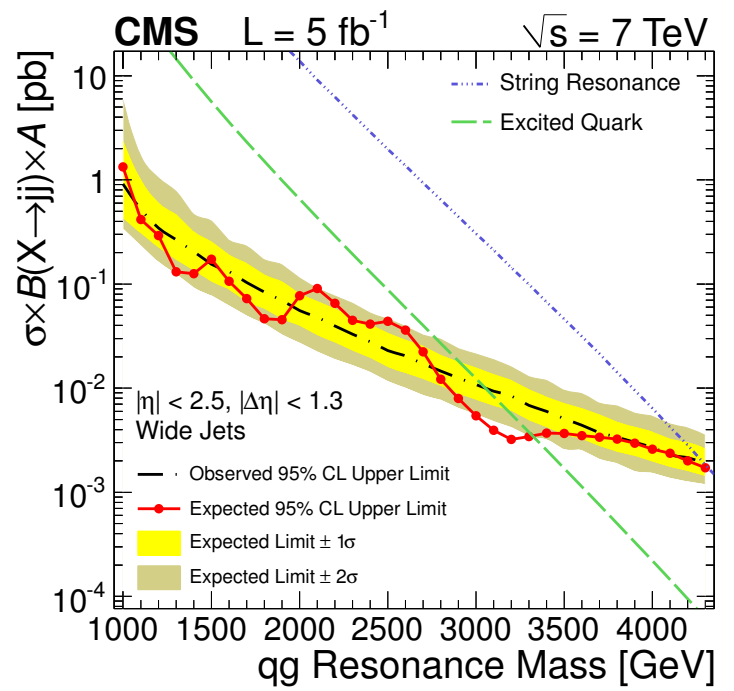

(b)

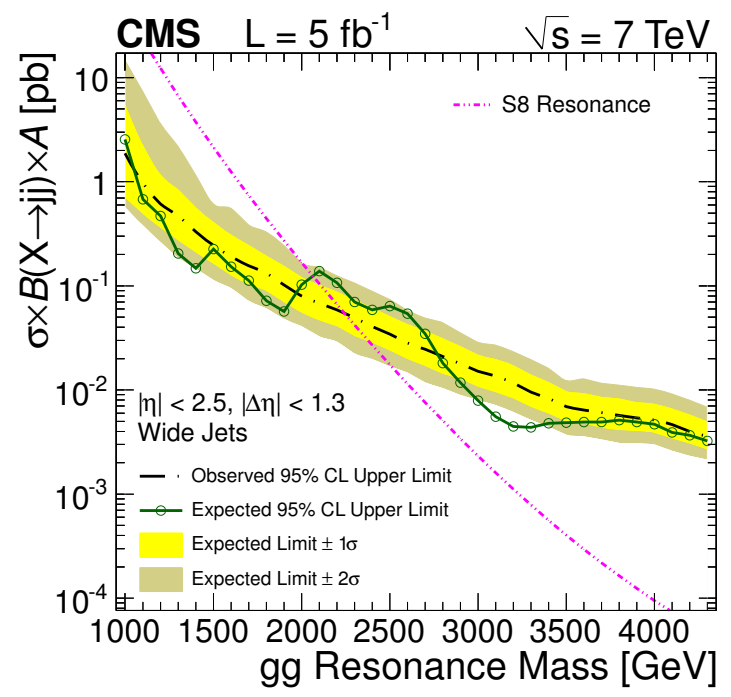

(c)

Figure 4. Observed 95\% CL upper limits on $\sigma \times B \times A$ for (a) quark-quark, (b) quark-gluon, (c) and gluon-gluon dijet resonances (points) from the inclusive analysis are compared to the expected limits (dot-dashed) and their variation at $1 \sigma$ and $2 \sigma$ levels (shaded bands). Theoretical predictions for various resonance models are also shown.

sion at $3.52 \mathrm{TeV}[20,21,25]$. For axigluons or colorons, we exclude masses smaller than $3.36 \mathrm{TeV}$; this extends our previous exclusion of $0.50<M(\mathrm{~A}, \mathrm{C})<2.47 \mathrm{TeV}[20,21]$ and is similar to the ATLAS limit of $3.32 \mathrm{TeV}$ based on $1 \mathrm{fb}^{-1}$ of data [24]. (We note here that the ATLAS and CMS experiments use different methods to calculate the axigluon and coloron cross section, which results in noticeable differences in the expected and observed mass limits for these models [25].) 


\begin{tabular}{|c|c|c|c|}
\hline Model & Final State & $\begin{array}{c}\text { Exp. Mass Exclusion } \\
{[\mathrm{TeV}]}\end{array}$ & $\begin{array}{c}\text { Obs. Mass Exclusion } \\
{[\mathrm{TeV}]}\end{array}$ \\
\hline String Resonance $(\mathrm{S})$ & $\mathrm{qg}$ & {$[1.0,4.29]$} & {$[1.0,4.31]$} \\
Excited Quark (q $\left.{ }^{*}\right)$ & $\mathrm{qg}$ & {$[1.0,3.05]$} & {$[1.0,3.32]$} \\
$\mathrm{E}_{6}$ Diquark $(\mathrm{D})$ & $\mathrm{qq}$ & {$[1.0,3.74]$} & {$[1.0,3.75]$} \\
Axigluon (A)/ Coloron (C) & $\mathrm{q} \overline{\mathrm{q}}$ & {$[1.0,3.16]$} & {$[1.0,3.36]$} \\
S8 Resonance (S8) & $\mathrm{gg}$ & {$[1.0,2.24]$} & {$[1.0,2.07]$} \\
$\mathrm{W}^{\prime}$ Boson $\left(\mathrm{W}^{\prime}\right)$ & $\mathrm{q} \overline{\mathrm{q}}$ & {$[1.0,1.78]$} & {$[1.0,1.92]$} \\
$\mathrm{Z}^{\prime}$ Boson $\left(\mathrm{Z}^{\prime}\right)$ & $\mathrm{q} \overline{\mathrm{q}}$ & {$[1.0,1.45]$} & {$[1.0,1.47]$} \\
\hline
\end{tabular}

Table 2. Observed and expected 95\% CL mass exclusions for specific models of dijet resonances from the inclusive analysis.

For the S8 color-octet model, we exclude masses in the range $1.0<M(\mathrm{~S} 8)<2.07 \mathrm{TeV}$; this extends the previous ATLAS exclusion of $0.9<M(\mathrm{~S} 8)<1.92 \mathrm{TeV}[24]$. For $\mathrm{W}^{\prime}$ bosons, we exclude masses in the range $1.00<M\left(\mathrm{~W}^{\prime}\right)<1.92 \mathrm{TeV}$; this extends the previous CMS exclusion limit $1.0<M\left(\mathrm{~W}^{\prime}\right)<1.51 \mathrm{TeV}[20,21]$. Finally, we exclude $\mathrm{Z}^{\prime}$ bosons in the mass range $1.0<M\left(\mathrm{Z}^{\prime}\right)<1.47 \mathrm{TeV}$. The observed and expected mass exclusions for specific models of dijet resonances are summarized in table 2 and are in generally good agreeement.

With the present data set, we start to be sensitive to the Randall-Sundrum gravitons just above $1 \mathrm{TeV}$ of mass. For the specific case of the Randall-Sundrum graviton, which couples either to a pair of gluons or to a quark-antiquark pair, the model-dependent limits on cross section are derived using a weighted average of the q $\bar{q}$ and gg dijet mass shapes, where the weights correspond to the relative branching fractions for these two final states. Although not strictly correct, approximate limits can be obtained by defining the modeldependent limits as a weighted average of the model-independent qq and gg limits. In the case of the Randall-Sundrum graviton, this approximate procedure was found to produce upper limits that differ by as much as $20 \%$ from those obtained using the weighted dijet mass shapes. However, for steeply falling signal cross sections, this difference would result in a relatively modest difference in the mass limit.

The 95\% CL observed upper limits on $\sigma \times B \times A$ for quantum black holes, derived from the inclusive analysis, are shown in figure 5 and reported in table 3 . The corresponding lower limits on the minimum mass of quantum black holes range from 4 to $5.3 \mathrm{TeV}$, depending on the model parameters, and are shown in figure 6 as a function of $M_{\mathrm{D}}$. These limits are slightly better than those obtained in ref. [19], where the same models were used. In ref. [19], a $S_{\mathrm{T}}$ variable, defined as $S_{\mathrm{T}}=\sum p_{\mathrm{T}}+E_{\mathrm{T}}^{\text {miss }}$ where the sum runs over individual objects: jets, electrons, photons, and muons, was used as a discriminator between the signal and the background, and counting experiments were performed above certain $S_{\mathrm{T}}$ values. In this analysis we take advantage of the fact that the shape of the signal in the dijet mass distribution is narrower than that in the generic $S_{\mathrm{T}}$ variable. This improved signal resolution allows us to extend the limits from the previous search. With the present data set, this analysis is not yet sensitive to the production of quantum black holes with $M_{\mathrm{D}}=5 \mathrm{TeV}$ and would require a factor of 2-3 increase in data to become sensitive to scenarios with $n=5-6$. 


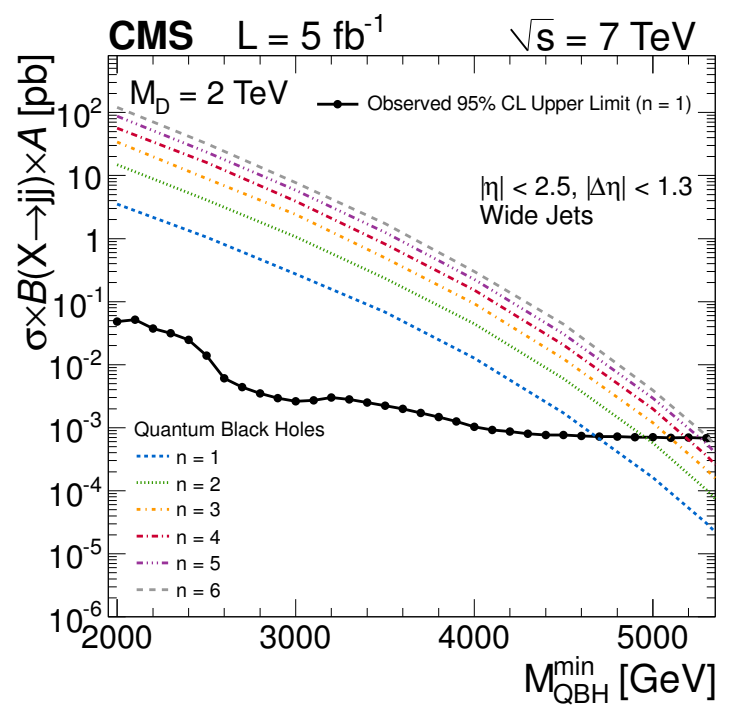

(a)

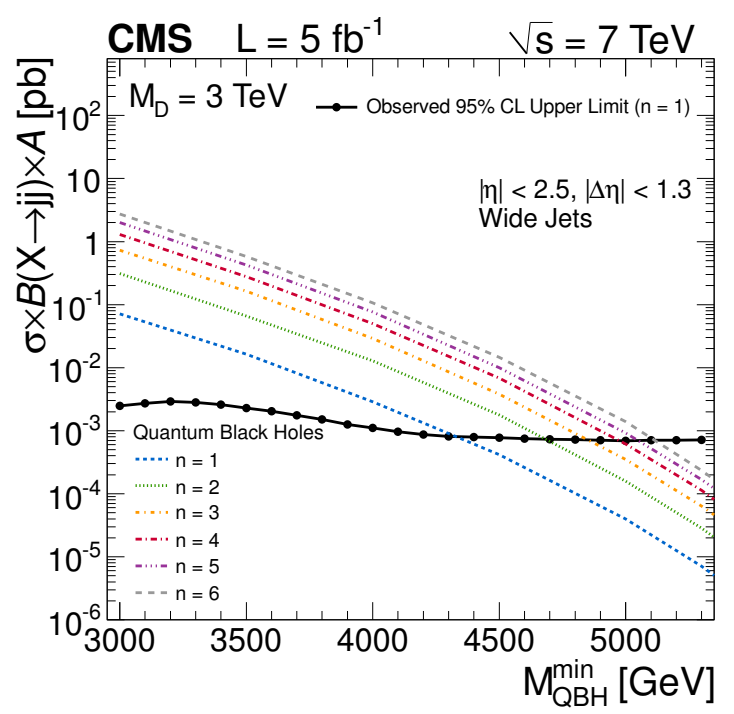

(b)

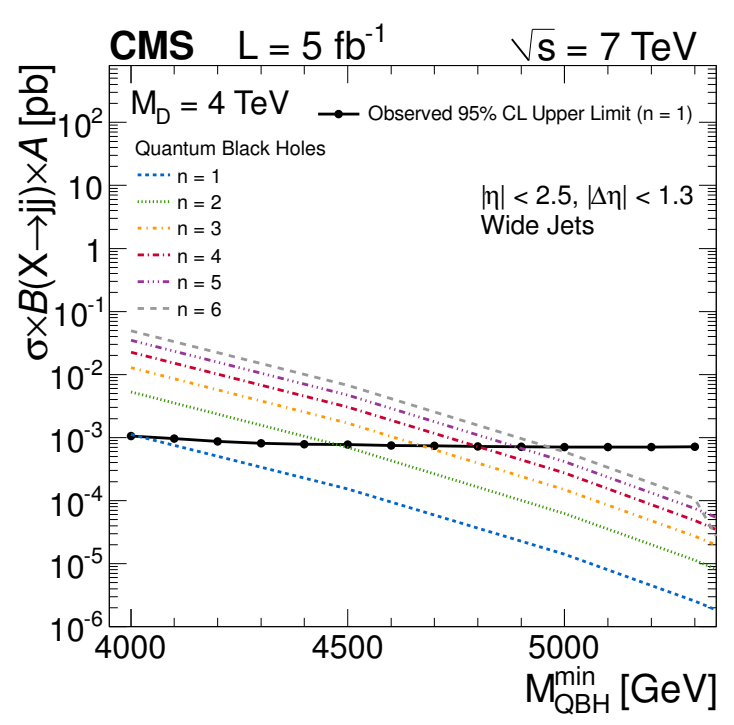

(c)

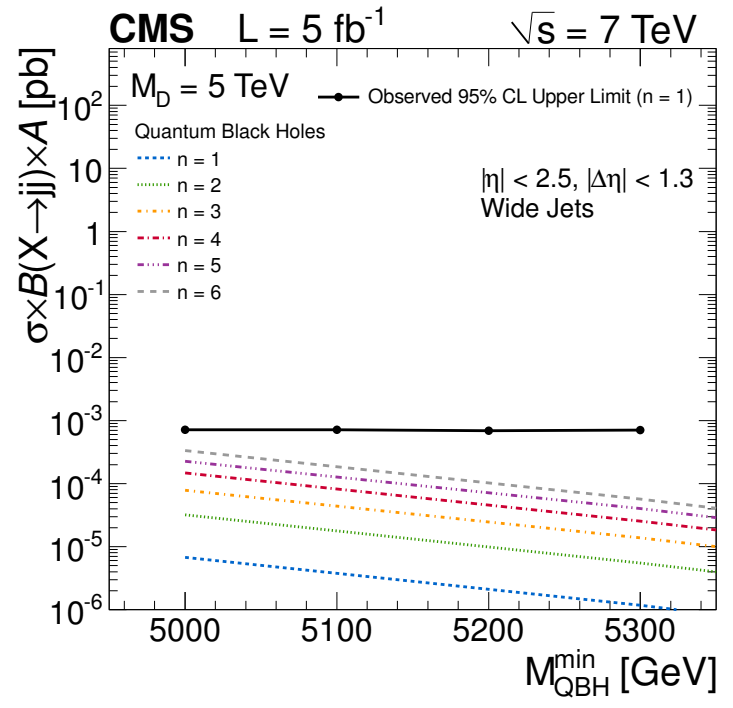

(d)

Figure 5. Observed $95 \% \mathrm{CL}$ upper limits on $\sigma \times B \times A$ as a function of the minimum mass of quantum black holes, compared to theoretical predictions for a quantum gravity scale of (a) $M_{\mathrm{D}}=2 \mathrm{TeV}$, (b) $M_{\mathrm{D}}=3 \mathrm{TeV}$, (c) $M_{\mathrm{D}}=4 \mathrm{TeV}$, and (d) $M_{\mathrm{D}}=5 \mathrm{TeV}$, with the number of extra dimensions $n$ ranging from one to six. The observed upper cross section limits are fairly independent of $n$ (limits for $n=2-6$ are within $\sim 5 \%$ of those for $n=1$ ) and would be practically indistinguishable in the above plots; therefore, for display purposes, only the observed upper limits for $n=1$ are shown. 


\begin{tabular}{|c|c|c|c|c|}
\hline \multirow{2}{*}{$\begin{array}{c}M_{\mathrm{QBH}}^{\min } \\
{[\mathrm{TeV}]}\end{array}$} & \multicolumn{4}{|c|}{ Upper limit on $\sigma \times B \times A[\mathrm{pb}]$} \\
\hline & $M_{\mathrm{D}}=2 \mathrm{TeV}$ & $M_{\mathrm{D}}=3 \mathrm{TeV}$ & $M_{\mathrm{D}}=4 \mathrm{TeV}$ & $M_{\mathrm{D}}=5 \mathrm{TeV}$ \\
\hline 2.0 & 0.048 & & & \\
\hline 2.1 & 0.051 & & & \\
\hline 2.2 & 0.037 & & & \\
\hline 2.3 & 0.032 & & & \\
\hline 2.4 & 0.025 & & & \\
\hline 2.5 & 0.014 & & & \\
\hline 2.6 & 0.0061 & & & \\
\hline 2.7 & 0.0044 & & & \\
\hline 2.8 & 0.0035 & & & \\
\hline 2.9 & 0.0029 & & & \\
\hline 3.0 & 0.0026 & 0.0025 & & \\
\hline 3.1 & 0.0027 & 0.0027 & & \\
\hline 3.2 & 0.0030 & 0.0029 & & \\
\hline 3.3 & 0.0028 & 0.0028 & & \\
\hline 3.4 & 0.0025 & 0.0026 & & \\
\hline 3.5 & 0.0022 & 0.0023 & & \\
\hline 3.6 & 0.0020 & 0.0020 & & \\
\hline 3.7 & 0.0017 & 0.0018 & & \\
\hline 3.8 & 0.0015 & 0.0015 & & \\
\hline 3.9 & 0.0013 & 0.0013 & & \\
\hline 4.0 & 0.0010 & 0.0011 & 0.0011 & \\
\hline 4.1 & 0.00092 & 0.00096 & 0.00096 & \\
\hline 4.2 & 0.00087 & 0.00087 & 0.00087 & \\
\hline 4.3 & 0.00080 & 0.00081 & 0.00081 & \\
\hline 4.4 & 0.00077 & 0.00079 & 0.00078 & \\
\hline 4.5 & 0.00076 & 0.00077 & 0.00077 & \\
\hline 4.6 & 0.00074 & 0.00075 & 0.00075 & \\
\hline 4.7 & 0.00072 & 0.00073 & 0.00074 & \\
\hline 4.8 & 0.00072 & 0.00072 & 0.00072 & \\
\hline 4.9 & 0.00071 & 0.00071 & 0.00071 & \\
\hline 5.0 & 0.00071 & 0.00069 & 0.00071 & 0.00071 \\
\hline 5.1 & 0.00069 & 0.00070 & 0.00071 & 0.00071 \\
\hline 5.2 & 0.00070 & 0.00071 & 0.00070 & 0.00069 \\
\hline 5.3 & 0.00068 & 0.00072 & 0.00071 & 0.00071 \\
\hline
\end{tabular}

Table 3. Observed 95\% CL upper limits on $\sigma \times B \times A$ for quantum black holes from the inclusive analysis. Only the limits for $n=1$ are reported. The limits for $n=2-6$ are within $\sim 5 \%$ of those for $n=1$. 


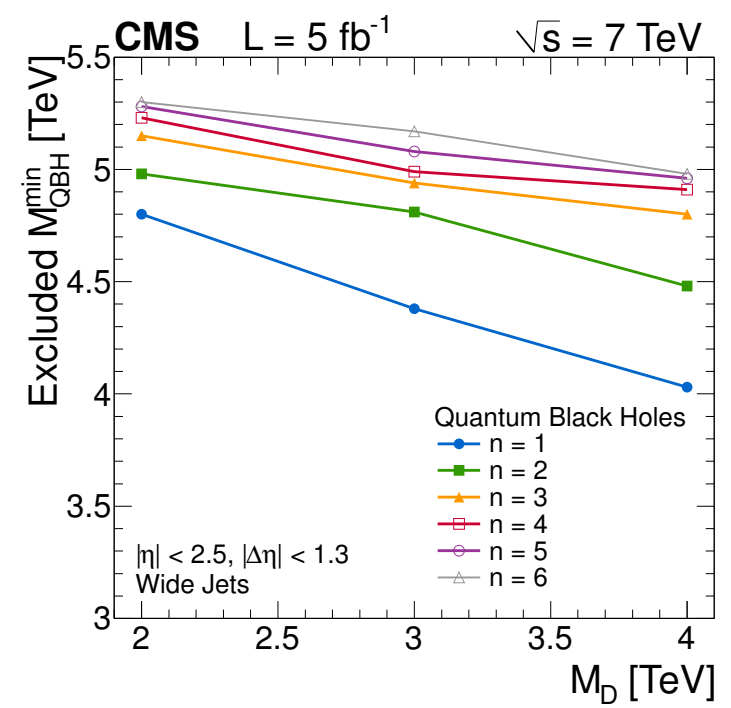

Figure 6. Observed 95\% CL lower limits on the minimum mass of quantum black holes as a function of the quantum gravity scale $M_{\mathrm{D}}$ for the number of extra dimensions $n$ of one (RandallSundrum model) and two to six (ADD model).

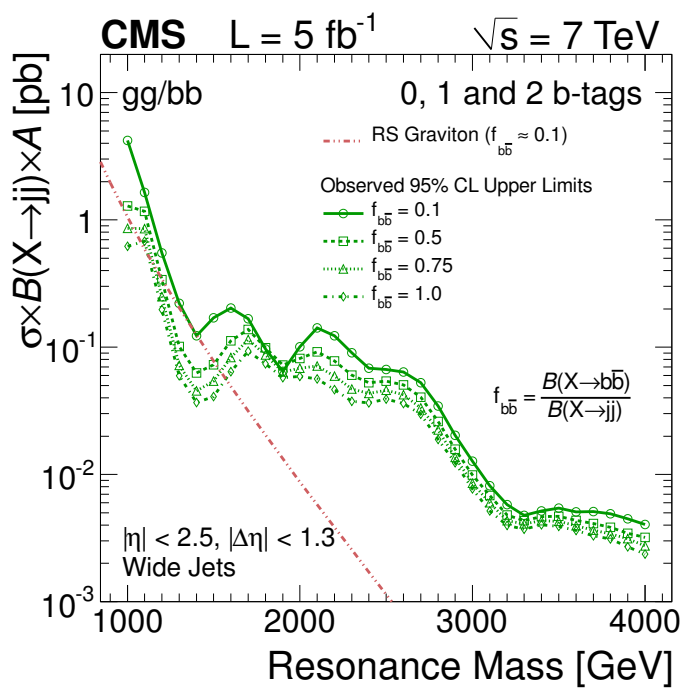

(a)

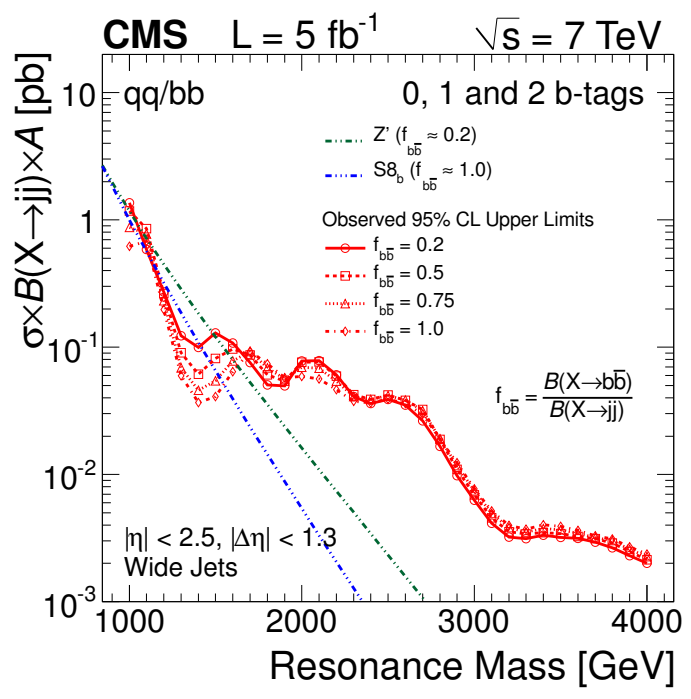

(b)

Figure 7. Observed 95\% CL upper limits on $\sigma \times B \times A$ for dijet resonances of type (a) gg/bb and (b) qq/bb, as defined in section 5.1, from the b-tagged analysis for four different values of $f_{\mathrm{b} \overline{\mathrm{b}}}$, compared to theoretical predictions for RS gravitons [11], $\mathrm{Z}^{\prime}$ bosons [10], and $\mathrm{S} 8_{\mathrm{b}}$ resonances [9].

Figure 7 shows the observed upper limits at the $95 \% \mathrm{CL}$ on $\sigma \times B \times A$ for $\mathrm{gg} / \mathrm{bb}$ and $\mathrm{qq} / \mathrm{bb}$ resonances from the b-tagged analysis for different values of $f_{\mathrm{b} \overline{\mathrm{b}}}$. For any model with known value of $f_{\mathrm{b} \bar{b}}$, the prediction of $\sigma \times B \times A$ at the parton level has to be compared to an appropriate limit curve in order to determine mass limits. The prediction for RS gravitons should be compared to the $f_{\mathrm{b} \overline{\mathrm{b}}}=0.1$ limit curve, for $\mathrm{Z}^{\prime}$ bosons to the $f_{\mathrm{b} \overline{\mathrm{b}}}=0.2$ 


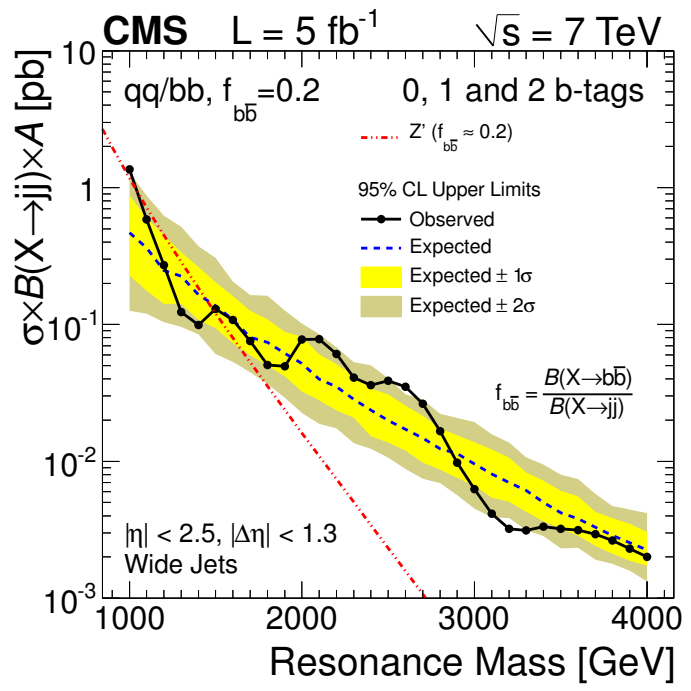

(a)

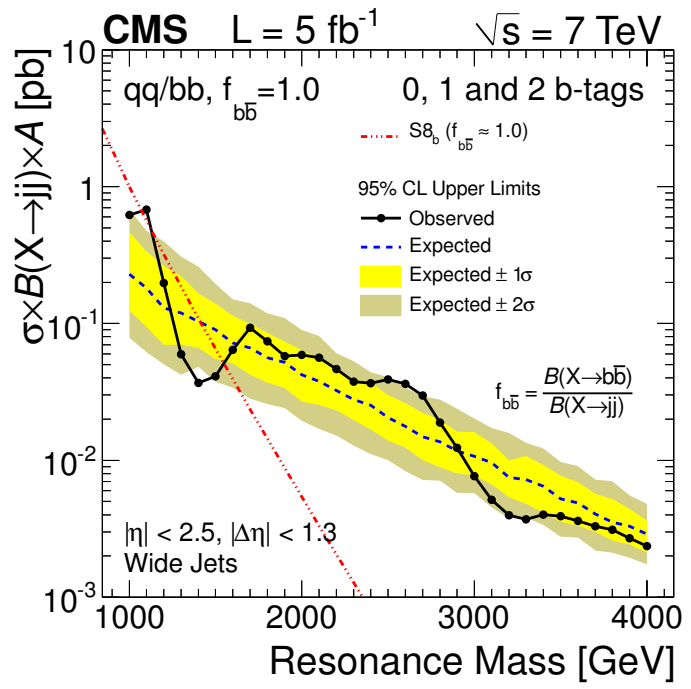

(b)

Figure 8. Observed 95\% CL upper limits on $\sigma \times B \times A$ for qq/bb resonances, as defined in section 5.1, from the b-tagged analysis with (a) $f_{\mathrm{b} \overline{\mathrm{b}}}=0.2$ and (b) $f_{\mathrm{b} \overline{\mathrm{b}}}=1.0$ (points), compared to the expected limits (dashed) and their variation at $1 \sigma$ and $2 \sigma$ levels (shaded bands). Theoretical predictions for $\mathrm{Z}^{\prime}$ bosons and $\mathrm{S} 8_{\mathrm{b}}$ resonances are also shown.

limit curve, and for $\mathrm{S} 8_{\mathrm{b}}$ resonances to the $f_{\mathrm{b} \overline{\mathrm{b}}}=1.0$ limit curve. The observed upper limits for signal masses between 1.0 and $4.0 \mathrm{TeV}$ and the values of $f_{\mathrm{b} \overline{\mathrm{b}}}$ shown in figure 7 are reported in table 4 . It is worth noting that for $\mathrm{gg} / \mathrm{bb}$ resonances, the limits become more stringent as $f_{\mathrm{b} \overline{\mathrm{b}}}$ increases. For example, for $\mathrm{gg} / \mathrm{bb}$ resonances with masses below $2 \mathrm{TeV}$, the upper cross section limits are as much as $70 \%$ lower for $f_{\mathrm{b} \overline{\mathrm{b}}}=1.0$ than for $f_{\mathrm{b} \overline{\mathrm{b}}}=0.1$. For $\mathrm{qq} / \mathrm{bb}$ resonances, however, this trend reverses at large values of the resonance mass since $\mathrm{b}$ tagging starts to lose its discriminating power and qq/bb mass shapes become wider as $f_{\mathrm{b} \overline{\mathrm{b}}}$ increases.

Figure 8 shows the expected limits and their uncertainty bands for qq/bb resonances with $f_{\mathrm{b} \overline{\mathrm{b}}}=0.2$ and $f_{\mathrm{b} \overline{\mathrm{b}}}=1.0$ and compares them to both the observed limits and theoretical predictions for $\mathrm{Z}^{\prime}$ bosons and $\mathrm{S} 8_{\mathrm{b}}$ resonances. The expected exclusion for $\mathrm{Z}^{\prime}$ bosons is $1.0<M\left(\mathrm{Z}^{\prime}\right)<1.45 \mathrm{TeV}$, and we exclude $1.04<M\left(\mathrm{Z}^{\prime}\right)<1.49 \mathrm{TeV}$. For $\mathrm{S} 8_{\mathrm{b}}$ resonances, the expected exclusion is $1.0<M\left(\mathrm{~S}_{\mathrm{b}}\right)<1.42 \mathrm{TeV}$, and we exclude $1.0<M\left(\mathrm{~S} 8_{\mathrm{b}}\right)<1.08 \mathrm{TeV}$ and $1.12<M\left(\mathrm{~S}_{\mathrm{b}}\right)<1.56 \mathrm{TeV}$. With the present data, no limits are set on the RS graviton mass. The observed and expected mass exclusions from the b-tagged analysis for $\mathrm{Z}^{\prime}$ bosons and $\mathrm{S} 8_{\mathrm{b}}$ resonances are summarized in table 5 .

\section{Summary}

A search for narrow resonances and quantum black holes in the dijet mass spectra has been performed using pp collisions at $\sqrt{s}=7 \mathrm{TeV}$, collected by the CMS detector at the LHC. Measured dijet mass spectra with and without b-tagging requirements are observed 


\begin{tabular}{|c|c|c|c|c|c|c|c|}
\hline \multirow{3}{*}{$\begin{array}{l}\text { Mass } \\
{[\mathrm{TeV}]}\end{array}$} & \multicolumn{7}{|c|}{ Upper limit on $\sigma \times B \times A[\mathrm{pb}]$} \\
\hline & \multicolumn{3}{|c|}{$\mathrm{gg} / \mathrm{bb}$} & \multicolumn{3}{|c|}{$\mathrm{qq} / \mathrm{bb}$} & \multirow{2}{*}{$\begin{array}{c}\mathrm{gg} / \mathrm{bb}, \mathrm{qq} / \mathrm{bb} \\
f_{\mathrm{b} \overline{\mathrm{b}}}=1.0\end{array}$} \\
\hline & $f_{\mathrm{b} \overline{\mathrm{b}}}=0.1$ & $f_{\mathrm{b} \overline{\mathrm{b}}}=0.5$ & $f_{\mathrm{b} \overline{\mathrm{b}}}=0.75$ & $f_{\mathrm{b} \overline{\mathrm{b}}}=0.2$ & $f_{\mathrm{b} \overline{\mathrm{b}}}=0.5$ & $f_{\mathrm{b} \overline{\mathrm{b}}}=0.75$ & \\
\hline 1.0 & 4.2 & 1.3 & 0.86 & 1.4 & 1.2 & 0.87 & 0.62 \\
\hline 1.1 & 1.6 & 1.2 & 0.85 & 0.59 & 0.85 & 0.78 & 0.68 \\
\hline 1.2 & 0.55 & 0.34 & 0.25 & 0.27 & 0.26 & 0.23 & 0.20 \\
\hline 1.3 & 0.22 & 0.10 & 0.072 & 0.12 & 0.09 & 0.069 & 0.060 \\
\hline 1.4 & 0.12 & 0.063 & 0.045 & 0.099 & 0.061 & 0.046 & 0.037 \\
\hline 1.5 & 0.17 & 0.073 & 0.054 & 0.13 & 0.082 & 0.055 & 0.041 \\
\hline 1.6 & 0.20 & 0.11 & 0.083 & 0.11 & 0.097 & 0.078 & 0.064 \\
\hline 1.7 & 0.17 & 0.14 & 0.11 & 0.076 & 0.087 & 0.093 & 0.093 \\
\hline 1.8 & 0.096 & 0.099 & 0.087 & 0.051 & 0.061 & 0.069 & 0.074 \\
\hline 1.9 & 0.065 & 0.072 & 0.064 & 0.050 & 0.054 & 0.056 & 0.058 \\
\hline 2.0 & 0.10 & 0.082 & 0.068 & 0.078 & 0.076 & 0.069 & 0.059 \\
\hline 2.1 & 0.14 & 0.092 & 0.071 & 0.078 & 0.077 & 0.068 & 0.056 \\
\hline 2.2 & 0.12 & 0.078 & 0.059 & 0.061 & 0.059 & 0.054 & 0.046 \\
\hline 2.3 & 0.09 & 0.060 & 0.047 & 0.041 & 0.042 & 0.041 & 0.038 \\
\hline 2.4 & 0.068 & 0.052 & 0.043 & 0.036 & 0.039 & 0.039 & 0.037 \\
\hline 2.5 & 0.067 & 0.054 & 0.046 & 0.039 & 0.042 & 0.042 & 0.039 \\
\hline 2.6 & 0.064 & 0.050 & 0.042 & 0.035 & 0.038 & 0.038 & 0.036 \\
\hline 2.7 & 0.053 & 0.040 & 0.033 & 0.026 & 0.032 & 0.032 & 0.030 \\
\hline 2.8 & 0.035 & 0.026 & 0.022 & 0.017 & 0.019 & 0.019 & 0.019 \\
\hline 2.9 & 0.020 & 0.016 & 0.014 & 0.0098 & 0.011 & 0.012 & 0.012 \\
\hline 3.0 & 0.013 & 0.0099 & 0.0085 & 0.0063 & 0.0069 & 0.0075 & 0.0077 \\
\hline 3.1 & 0.0082 & 0.0068 & 0.0060 & 0.0041 & 0.0046 & 0.0049 & 0.0052 \\
\hline 3.2 & 0.0058 & 0.0048 & 0.0043 & 0.0032 & 0.0036 & 0.0038 & 0.0040 \\
\hline 3.3 & 0.0048 & 0.0043 & 0.0041 & 0.0031 & 0.0034 & 0.0035 & 0.0037 \\
\hline 3.4 & 0.0052 & 0.0047 & 0.0043 & 0.0033 & 0.0035 & 0.0037 & 0.0040 \\
\hline 3.5 & 0.0054 & 0.0047 & 0.0043 & 0.0032 & 0.0034 & 0.0037 & 0.0039 \\
\hline 3.6 & 0.0051 & 0.0043 & 0.0040 & 0.0031 & 0.0033 & 0.0034 & 0.0036 \\
\hline 3.7 & 0.0051 & 0.0041 & 0.0037 & 0.0029 & 0.0031 & 0.0032 & 0.0033 \\
\hline 3.8 & 0.0049 & 0.0038 & 0.0034 & 0.0026 & 0.0029 & 0.0030 & 0.0031 \\
\hline 3.9 & 0.0045 & 0.0034 & 0.0031 & 0.0023 & 0.0025 & 0.0026 & 0.0027 \\
\hline 4.0 & 0.0041 & 0.0032 & 0.0027 & 0.0020 & 0.0021 & 0.0023 & 0.0024 \\
\hline
\end{tabular}

Table 4. Observed 95\% CL upper limits on $\sigma \times B \times A$ for narrow $\mathrm{gg} / \mathrm{bb}$ and $\mathrm{qq} / \mathrm{bb}$ resonances, as defined in section 5.1, from the b-tagged analysis for signal masses between 1.0 and $4.0 \mathrm{TeV}$.

to be consistent with the standard model expectation of a smoothly falling distribution. There is no evidence for new particle production in the data. Model-independent upper limits are presented on the product $\sigma \times B \times A$ that are applicable to any model of narrow dijet resonance production, and with $\mathrm{b}$ tagging applied, limits are expressed in terms of the branching fraction to b-jet pairs. Lower limits are obtained on the mass of string resonances, 


\begin{tabular}{|c|c|c|c|}
\hline Model & Final State & $\begin{array}{c}\text { Exp. Mass Exclusion } \\
{[\mathrm{TeV}]}\end{array}$ & $\begin{array}{c}\text { Obs. Mass Exclusion } \\
{[\mathrm{TeV}]}\end{array}$ \\
\hline $\mathrm{Z}^{\prime}$ Boson $\left(\mathrm{Z}^{\prime}\right)$ & $\mathrm{q} \overline{\mathrm{q}}$ & {$[1.0,1.45]$} & {$[1.04,1.49]$} \\
$\mathrm{S} 8_{\mathrm{b}}$ Resonance $\left(\mathrm{S} 8_{\mathrm{b}}\right)$ & $\mathrm{q} \overline{\mathrm{q}}$ & {$[1.0,1.42]$} & {$[1.0,1.08],[1.12,1.56]$} \\
\hline
\end{tabular}

Table 5. Observed and expected 95\% CL mass exclusions from the b-tagged analysis for $\mathrm{Z}^{\prime}$ bosons and $\mathrm{S} 8_{\mathrm{b}}$ resonances.

excited quarks, axigluons and colorons, scalar color-octet resonances, $\mathrm{E}_{6}$ diquarks, $\mathrm{W}^{\prime}$ and $\mathrm{Z}^{\prime}$ bosons, and quantum black holes. Most of these limits extend excluded mass ranges from the previous searches.

\section{Acknowledgments}

We thank Can Kilic for calculations of the string resonance cross section, Ian Lewis for calculations of the S8 model cross section, and Bogdan Dobrescu for assistance in implementing the $\mathrm{S} 8_{\mathrm{b}}$ model. We congratulate our colleagues in the CERN accelerator departments for the excellent performance of the LHC machine. We thank the technical and administrative staff at CERN and other CMS institutes, and acknowledge support from: BMWF and FWF (Austria); FNRS and FWO (Belgium); CNPq, CAPES, FAPERJ, and FAPESP (Brazil); MES (Bulgaria); CERN; CAS, MoST, and NSFC (China); COLCIENCIAS (Colombia); MSES (Croatia); RPF (Cyprus); MoER, SF0690030s09 and ERDF (Estonia); Academy of Finland, MEC, and HIP (Finland); CEA and CNRS/IN2P3 (France); BMBF, DFG, and HGF (Germany); GSRT (Greece); OTKA and NKTH (Hungary); DAE and DST (India); IPM (Iran); SFI (Ireland); INFN (Italy); NRF and WCU (Korea); LAS (Lithuania); CINVESTAV, CONACYT, SEP, and UASLP-FAI (Mexico); MSI (New Zealand); PAEC (Pakistan); MSHE and NSC (Poland); FCT (Portugal); JINR (Armenia, Belarus, Georgia, Ukraine, Uzbekistan); MON, RosAtom, RAS and RFBr (Russia); MSTD (Serbia); SEIDI and CPAN (Spain); Swiss Funding Agencies (Switzerland); NSC (Taipei); TUBITAK and TAEK (Turkey); STFC (United Kingdom); DOE and NSF (U.S.A.).

Open Access. This article is distributed under the terms of the Creative Commons Attribution License which permits any use, distribution and reproduction in any medium, provided the original author(s) and source are credited.

\section{References}

[1] L.A. Anchordoqui, H. Goldberg, S. Nawata and T.R. Taylor, Jet signals for low mass strings at the LHC, Phys. Rev. Lett. 100 (2008) 171603 [arXiv:0712.0386] [InSPIRE].

[2] S. Cullen, M. Perelstein and M.E. Peskin, TeV strings and collider probes of large extra dimensions, Phys. Rev. D 62 (2000) 055012 [hep-ph/0001166] [INSPIRE].

[3] J.L. Hewett and T.G. Rizzo, Low-Energy Phenomenology of Superstring Inspired E 6 Models, Phys. Rept. 183 (1989) 193 [INSPIRE]. 
[4] U. Baur, I. Hinchliffe and D. Zeppenfeld, Excited Quark Production at Hadron Colliders, Int. J. Mod. Phys. A 2 (1987) 1285 [inSPIRE].

[5] U. Baur, M. Spira and P. Zerwas, Excited quark and lepton production at hadron colliders, Phys. Rev. D 42 (1990) 815 [inSPIRE].

[6] P.H. Frampton and S.L. Glashow, Chiral Color: An Alternative to the Standard Model, Phys. Lett. B 190 (1987) 157 [inSPIRE].

[7] E.H. Simmons, Coloron phenomenology, Phys. Rev. D 55 (1997) 1678 [hep-ph/9608269] [INSPIRE].

[8] T. Han, I. Lewis and Z. Liu, Colored Resonant Signals at the LHC: Largest Rate and Simplest Topology, JHEP 12 (2010) 085 [arXiv: 1010.4309] [INSPIRE].

[9] Y. Bai and B.A. Dobrescu, Heavy octets and Tevatron signals with three or four b jets, JHEP 07 (2011) 100 [arXiv: 1012.5814] [INSPIRE].

[10] E. Eichten, I. Hinchliffe, K.D. Lane and C. Quigg, Super Collider Physics, Rev. Mod. Phys. 56 (1984) 579 [Addendum ibid. 58 (1986) 1065] [INSPIRE].

[11] L. Randall and R. Sundrum, An Alternative to compactification, Phys. Rev. Lett. 83 (1999) 4690 [hep-th/9906064] [INSPIRE].

[12] B. Allanach et al., Exploring small extra dimensions at the large hadron collider, JHEP 12 (2002) 039 [hep-ph/0211205] [INSPIRE].

[13] P. Meade and L. Randall, Black Holes and Quantum Gravity at the LHC, JHEP 05 (2008) 003 [arXiv:0708.3017] [INSPIRE].

[14] X. Calmet, W. Gong and S.D. Hsu, Colorful quantum black holes at the LHC, Phys. Lett. B 668 (2008) 20 [arXiv:0806.4605] [InSPIRE].

[15] D.M. Gingrich, Quantum black holes with charge, colour and spin at the LHC, J. Phys. G 37 (2010) 105008 [arXiv:0912.0826] [INSPIRE].

[16] N. Arkani-Hamed, S. Dimopoulos and G. Dvali, The Hierarchy problem and new dimensions at a millimeter, Phys. Lett. B 429 (1998) 263 [hep-ph/9803315] [INSPIRE].

[17] N. Arkani-Hamed, S. Dimopoulos and G. Dvali, Phenomenology, astrophysics and cosmology of theories with submillimeter dimensions and TeV scale quantum gravity, Phys. Rev. D 59 (1999) 086004 [hep-ph/9807344] [INSPIRE].

[18] L. Randall and R. Sundrum, A Large mass hierarchy from a small extra dimension, Phys. Rev. Lett. 83 (1999) 3370 [hep-ph/9905221] [INSPIRE].

[19] CMS collaboration, Search for microscopic black holes in pp collisions at $\sqrt{s}=7$ TeV, JHEP 04 (2012) 061 [arXiv: 1202.6396] [INSPIRE].

[20] CMS collaboration, Search for Dijet Resonances in 7 TeV pp Collisions at CMS, Phys. Rev. Lett. 105 (2010) 211801 [arXiv:1010.0203] [INSPIRE].

[21] CMS collaboration, Search for Resonances in the Dijet Mass Spectrum from $7 \mathrm{TeV} p p$ Collisions at CMS, Phys. Lett. B 704 (2011) 123 [arXiv:1107.4771] [INSPIRE].

[22] ATLAS collaboration, Search for New Particles in Two-Jet Final States in $7 \mathrm{TeV}$ Proton-Proton Collisions with the ATLAS Detector at the LHC, Phys. Rev. Lett. 105 (2010) 161801 [arXiv: 1008.2461] [INSPIRE]. 
[23] ATLAS collaboration, Search for New Physics in Dijet Mass and Angular Distributions in pp Collisions at $\sqrt{s}=7$ TeV Measured with the ATLAS Detector, New J. Phys. 13 (2011) 053044 [arXiv: 1103.3864] [INSPIRE].

[24] ATLAS collaboration, Search for New Physics in the Dijet Mass Distribution using $1 \mathrm{fb}^{-1}$ of pp Collision Data at $\sqrt{s}=7 \mathrm{TeV}$ collected by the ATLAS Detector, Phys. Lett. B 708 (2012) 37 [arXiv: 1108.6311] [INSPIRE].

[25] R.M. Harris and K. Kousouris, Searches for Dijet Resonances at Hadron Colliders, Int. J. Mod. Phys. A 26 (2011) 5005 [arXiv:1110.5302] [INSPIRE].

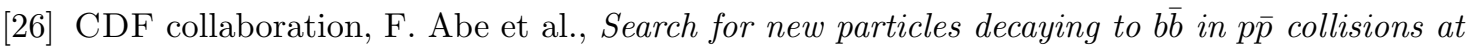
$\sqrt{s}=1.8 \mathrm{TeV}$, Phys. Rev. Lett. 82 (1999) 2038 [hep-ex/9809022] [INSPIRE].

[27] CMS collaboration, The CMS experiment at the CERN LHC, JINST 3 (2008) S08004 [INSPIRE].

[28] CMS collaboration, Absolute Calibration of the Luminosity Measurement at CMS: Winter 2012 Update, CMS-PAS-SMP-12-008.

[29] M. Cacciari, G.P. Salam and G. Soyez, The Anti-k $k_{t}$ jet clustering algorithm, JHEP 04 (2008) 063 [arXiv: 0802.1189] [INSPIRE].

[30] CMS collaboration, Particle-flow Event Reconstruction in CMS and Performance for Jets, Taus, and MET, CMS-PAS-PFT-09-001.

[31] CMS collaboration, Commissioning of the Particle-flow Event Reconstruction with the First LHC Collisions Recorded in the CMS Detector, CMS-PAS-PFT-10-001.

[32] CMS collaboration, Jet Performance in pp Collisions at $\sqrt{s}=7 \mathrm{TeV}$, CMS-PAS-JME-10-003.

[33] CMS collaboration, Determination of Jet Energy Calibration and Transverse Momentum Resolution in CMS, 2011 JINST 6 P11002 [arXiv:1107.4277] [INSPIRE].

[34] M. Cacciari and G.P. Salam, Pileup subtraction using jet areas, Phys. Lett. B 659 (2008) 119 [arXiv: 0707.1378] [INSPIRE].

[35] M. Cacciari, J. Rojo, G.P. Salam and G. Soyez, Quantifying the performance of jet definitions for kinematic reconstruction at the LHC, JHEP 12 (2008) 032 [arXiv:0810.1304] [INSPIRE].

[36] CMS collaboration, b-jet Identification in the CMS Experiment, CMS-PAS-BTV-11-004.

[37] CDF collaboration, T. Aaltonen et al., Search for new particles decaying into dijets in proton-antiproton collisions at $\sqrt{s}=1.96$-TeV, Phys. Rev. D 79 (2009) 112002 [arXiv: 0812.4036] [INSPIRE].

[38] T. Sjöstrand, S. Mrenna and P.Z. Skands, PYTHIA 6.4 Physics and Manual, JHEP 05 (2006) 026 [hep-ph/0603175] [INSPIRE].

[39] GEANT4 collaboration, S. Agostinelli et al., GEANT4: A Simulation toolkit, Nucl. Instrum. Meth. A 506 (2003) 250 [inSPIRE].

[40] GEANT4 collaboration, J. Allison et al., Geant4 developments and applications, IEEE Trans. Nucl. Sci. 53 (2006) 270 [INSPIRE].

[41] J. Pumplin et al., New generation of parton distributions with uncertainties from global QCD analysis, JHEP 07 (2002) 012 [hep-ph/0201195] [INSPIRE]. 
[42] R. Field, Early LHC Underlying Event Data - Findings and Surprises, arXiv:1010.3558 [INSPIRE].

[43] S. Dimopoulos and G.L. Landsberg, Black holes at the LHC, Phys. Rev. Lett. 87 (2001) 161602 [hep-ph/0106295] [INSPIRE].

[44] S.B. Giddings and S.D. Thomas, High-energy colliders as black hole factories: The End of short distance physics, Phys. Rev. D 65 (2002) 056010 [hep-ph/0106219] [INSPIRE].

[45] D.M. Gingrich, Monte Carlo event generator for black hole production and decay in proton-proton collisions, Comput. Phys. Commun. 181 (2010) 1917 [arXiv:0911.5370] [INSPIRE].

[46] CMS collaboration, The fast simulation of the CMS detector at LHC, J. Phys. Conf. S. 331 (2011) 032049.

[47] Particle Data Group collaboration, J. Beringer et al., Review of Particle Physics (RPP), Phys. Rev. D 86 (2012) 010001 [inSPIRE].

[48] J. Alwall, M. Herquet, F. Maltoni, O. Mattelaer and T. Stelzer, MadGraph 5: Going Beyond, JHEP 06 (2011) 128 [arXiv:1106.0522] [INSPIRE].

[49] R.S. Chivukula, A. Farzinnia, E.H. Simmons and R. Foadi, Production of Massive Color-Octet Vector Bosons at Next-to-Leading Order, Phys. Rev. D 85 (2012) 054005 [arXiv:1111.7261] [INSPIRE]. 


\section{The CMS collaboration}

\section{Yerevan Physics Institute, Yerevan, Armenia}

S. Chatrchyan, V. Khachatryan, A.M. Sirunyan, A. Tumasyan

\section{Institut für Hochenergiephysik der OeAW, Wien, Austria}

W. Adam, E. Aguilo, T. Bergauer, M. Dragicevic, J. Erö, C. Fabjan ${ }^{1}$, M. Friedl, R. Frühwirth ${ }^{1}$, V.M. Ghete, N. Hörmann, J. Hrubec, M. Jeitler ${ }^{1}$, W. Kiesenhofer, V. Knünz, M. Krammer ${ }^{1}$, I. Krätschmer, D. Liko, I. Mikulec, M. Pernicka ${ }^{\dagger}$, D. Rabady ${ }^{2}$, B. Rahbaran, C. Rohringer, H. Rohringer, R. Schöfbeck, J. Strauss, A. Taurok, W. Waltenberger, C.-E. Wulz ${ }^{1}$

National Centre for Particle and High Energy Physics, Minsk, Belarus

V. Mossolov, N. Shumeiko, J. Suarez Gonzalez

\section{Universiteit Antwerpen, Antwerpen, Belgium}

S. Alderweireldt, M. Bansal, S. Bansal, T. Cornelis, E.A. De Wolf, X. Janssen, S. Luyckx, L. Mucibello, S. Ochesanu, B. Roland, R. Rougny, H. Van Haevermaet, P. Van Mechelen, N. Van Remortel, A. Van Spilbeeck

\section{Vrije Universiteit Brussel, Brussel, Belgium}

F. Blekman, S. Blyweert, J. D'Hondt, R. Gonzalez Suarez, A. Kalogeropoulos, M. Maes, A. Olbrechts, S. Tavernier, W. Van Doninck, P. Van Mulders, G.P. Van Onsem, I. Villella

Université Libre de Bruxelles, Bruxelles, Belgium

B. Clerbaux, G. De Lentdecker, V. Dero, A.P.R. Gay, T. Hreus, A. Léonard, P.E. Marage, A. Mohammadi, T. Reis, L. Thomas, C. Vander Velde, P. Vanlaer, J. Wang

\section{Ghent University, Ghent, Belgium}

V. Adler, K. Beernaert, A. Cimmino, S. Costantini, G. Garcia, M. Grunewald, B. Klein, J. Lellouch, A. Marinov, J. Mccartin, A.A. Ocampo Rios, D. Ryckbosch, M. Sigamani, N. Strobbe, F. Thyssen, M. Tytgat, S. Walsh, E. Yazgan, N. Zaganidis

Université Catholique de Louvain, Louvain-la-Neuve, Belgium

S. Basegmez, G. Bruno, R. Castello, L. Ceard, C. Delaere, T. du Pree, D. Favart, L. Forthomme, A. Giammanco ${ }^{3}$, J. Hollar, V. Lemaitre, J. Liao, O. Militaru, C. Nuttens, D. Pagano, A. Pin, K. Piotrzkowski, M. Selvaggi, J.M. Vizan Garcia

Université de Mons, Mons, Belgium

N. Beliy, T. Caebergs, E. Daubie, G.H. Hammad

Centro Brasileiro de Pesquisas Fisicas, Rio de Janeiro, Brazil

G.A. Alves, M. Correa Martins Junior, T. Martins, M.E. Pol, M.H.G. Souza

Universidade do Estado do Rio de Janeiro, Rio de Janeiro, Brazil

W.L. Aldá Júnior, W. Carvalho, J. Chinellato ${ }^{4}$, A. Custódio, E.M. Da Costa, D. De Jesus Damiao, C. De Oliveira Martins, S. Fonseca De Souza, H. Malbouisson, M. Malek, D. Matos Figueiredo, L. Mundim, H. Nogima, W.L. Prado Da Silva, A. Santoro, L. Soares Jorge, A. Sznajder, E.J. Tonelli Manganote ${ }^{4}$, A. Vilela Pereira 
Instituto de Fisica Teorica ${ }^{a}$, Universidade Estadual Paulista ${ }^{b}$, Sao Paulo, Brazil

T.S. Anjos ${ }^{b, 5}$, C.A. Bernardes ${ }^{b, 5}$, F.A. Dias ${ }^{a, 6}$, T.R. Fernandez Perez Tomei ${ }^{a}$, E.M. Gregores ${ }^{b, 5}$, C. Lagana ${ }^{a}$, F. Marinho ${ }^{a}$, P.G. Mercadante ${ }^{b, 5}$, S.F. Novaes ${ }^{a}$, Sandra $^{2}$ S. Padula ${ }^{a}$

Institute for Nuclear Research and Nuclear Energy, Sofia, Bulgaria

V. Genchev ${ }^{2}$, P. Iaydjiev², S. Piperov, M. Rodozov, S. Stoykova, G. Sultanov, V. Tcholakov, R. Trayanov, M. Vutova

University of Sofia, Sofia, Bulgaria

A. Dimitrov, R. Hadjiiska, V. Kozhuharov, L. Litov, B. Pavlov, P. Petkov

Institute of High Energy Physics, Beijing, China

J.G. Bian, G.M. Chen, H.S. Chen, C.H. Jiang, D. Liang, S. Liang, X. Meng, J. Tao, J. Wang, X. Wang, Z. Wang, H. Xiao, M. Xu, J. Zang, Z. Zhang

State Key Lab. of Nucl. Phys. and Tech., Peking University, Beijing, China

C. Asawatangtrakuldee, Y. Ban, Y. Guo, W. Li, S. Liu, Y. Mao, S.J. Qian, H. Teng, D. Wang, L. Zhang, W. Zou

Universidad de Los Andes, Bogota, Colombia

C. Avila, C.A. Carrillo Montoya, J.P. Gomez, B. Gomez Moreno, A.F. Osorio Oliveros, J.C. Sanabria

Technical University of Split, Split, Croatia

N. Godinovic, D. Lelas, R. Plestina ${ }^{7}$, D. Polic, I. Puljak ${ }^{2}$

University of Split, Split, Croatia

Z. Antunovic, M. Kovac

Institute Rudjer Boskovic, Zagreb, Croatia

V. Brigljevic, S. Duric, K. Kadija, J. Luetic, D. Mekterovic, S. Morovic, L. Tikvica

University of Cyprus, Nicosia, Cyprus

A. Attikis, M. Galanti, G. Mavromanolakis, J. Mousa, C. Nicolaou, F. Ptochos, P.A. Razis

Charles University, Prague, Czech Republic

M. Finger, M. Finger Jr.

Academy of Scientific Research and Technology of the Arab Republic of Egypt, Egyptian Network of High Energy Physics, Cairo, Egypt

Y. Assran ${ }^{8}$, S. Elgammal ${ }^{9}$, A. Ellithi Kamel ${ }^{10}$, A.M. Kuotb Awad ${ }^{11}$, M.A. Mahmoud ${ }^{11}$, A. Radi ${ }^{12,13}$

National Institute of Chemical Physics and Biophysics, Tallinn, Estonia

M. Kadastik, M. Müntel, M. Murumaa, M. Raidal, L. Rebane, A. Tiko

Department of Physics, University of Helsinki, Helsinki, Finland

P. Eerola, G. Fedi, M. Voutilainen 


\section{Helsinki Institute of Physics, Helsinki, Finland}

J. Härkönen, A. Heikkinen, V. Karimäki, R. Kinnunen, M.J. Kortelainen, T. Lampén, K. Lassila-Perini, S. Lehti, T. Lindén, P. Luukka, T. Mäenpää, T. Peltola, E. Tuominen, J. Tuominiemi, E. Tuovinen, D. Ungaro, L. Wendland

\section{Lappeenranta University of Technology, Lappeenranta, Finland}

A. Korpela, T. Tuuva

DSM/IRFU, CEA/Saclay, Gif-sur-Yvette, France

M. Besancon, S. Choudhury, F. Couderc, M. Dejardin, D. Denegri, B. Fabbro, J.L. Faure, F. Ferri, S. Ganjour, A. Givernaud, P. Gras, G. Hamel de Monchenault, P. Jarry, E. Locci, J. Malcles, L. Millischer, A. Nayak, J. Rander, A. Rosowsky, M. Titov

Laboratoire Leprince-Ringuet, Ecole Polytechnique, IN2P3-CNRS, Palaiseau, France

S. Baffioni, F. Beaudette, L. Benhabib, L. Bianchini, M. Bluj ${ }^{14}$, P. Busson, C. Charlot,

N. Daci, T. Dahms, M. Dalchenko, L. Dobrzynski, A. Florent, R. Granier de Cassagnac, M. Haguenauer, P. Miné, C. Mironov, I.N. Naranjo, M. Nguyen, C. Ochando, P. Paganini, D. Sabes, R. Salerno, Y. Sirois, C. Veelken, A. Zabi

Institut Pluridisciplinaire Hubert Curien, Université de Strasbourg, Université de Haute Alsace Mulhouse, CNRS/IN2P3, Strasbourg, France J.-L. Agram ${ }^{15}$, J. Andrea, D. Bloch, D. Bodin, J.-M. Brom, M. Cardaci, E.C. Chabert, C. Collard, E. Conte ${ }^{15}$, F. Drouhin ${ }^{15}$, J.-C. Fontaine ${ }^{15}$, D. Gelé, U. Goerlach, P. Juillot, A.-C. Le Bihan, P. Van Hove

Université de Lyon, Université Claude Bernard Lyon 1, CNRS-IN2P3, Institut de Physique Nucléaire de Lyon, Villeurbanne, France

S. Beauceron, N. Beaupere, O. Bondu, G. Boudoul, S. Brochet, J. Chasserat, R. Chierici² D. Contardo, P. Depasse, H. El Mamouni, J. Fay, S. Gascon, M. Gouzevitch, B. Ille, T. Kurca, M. Lethuillier, L. Mirabito, S. Perries, L. Sgandurra, V. Sordini, Y. Tschudi, P. Verdier, S. Viret

Institute of High Energy Physics and Informatization, Tbilisi State University, Tbilisi, Georgia

Z. Tsamalaidze ${ }^{16}$

RWTH Aachen University, I. Physikalisches Institut, Aachen, Germany

C. Autermann, S. Beranek, B. Calpas, M. Edelhoff, L. Feld, N. Heracleous, O. Hindrichs, R. Jussen, K. Klein, J. Merz, A. Ostapchuk, A. Perieanu, F. Raupach, J. Sammet, S. Schael, D. Sprenger, H. Weber, B. Wittmer, V. Zhukov ${ }^{17}$

RWTH Aachen University, III. Physikalisches Institut A, Aachen, Germany M. Ata, J. Caudron, E. Dietz-Laursonn, D. Duchardt, M. Erdmann, R. Fischer, A. Güth, T. Hebbeker, C. Heidemann, K. Hoepfner, D. Klingebiel, P. Kreuzer, M. Merschmeyer, A. Meyer, M. Olschewski, K. Padeken, P. Papacz, H. Pieta, H. Reithler, S.A. Schmitz, L. Sonnenschein, J. Steggemann, D. Teyssier, S. Thüer, M. Weber 
RWTH Aachen University, III. Physikalisches Institut B, Aachen, Germany M. Bontenackels, V. Cherepanov, Y. Erdogan, G. Flügge, H. Geenen, M. Geisler, W. Haj Ahmad, F. Hoehle, B. Kargoll, T. Kress, Y. Kuessel, J. Lingemann², A. Nowack, I.M. Nugent, L. Perchalla, O. Pooth, P. Sauerland, A. Stahl

\section{Deutsches Elektronen-Synchrotron, Hamburg, Germany}

M. Aldaya Martin, I. Asin, N. Bartosik, J. Behr, W. Behrenhoff, U. Behrens, M. Bergholz ${ }^{18}$, A. Bethani, K. Borras, A. Burgmeier, A. Cakir, L. Calligaris, A. Campbell, E. Castro, F. Costanza, D. Dammann, C. Diez Pardos, T. Dorland, G. Eckerlin, D. Eckstein, G. Flucke, A. Geiser, I. Glushkov, P. Gunnellini, S. Habib, J. Hauk, G. Hellwig, H. Jung, M. Kasemann, P. Katsas, C. Kleinwort, H. Kluge, A. Knutsson, M. Krämer, D. Krücker, E. Kuznetsova, W. Lange, J. Leonard, W. Lohmann ${ }^{18}$, B. Lutz, R. Mankel, I. Marfin, M. Marienfeld, I.-A. Melzer-Pellmann, A.B. Meyer, J. Mnich, A. Mussgiller, S. Naumann-Emme, O. Novgorodova, F. Nowak, J. Olzem, H. Perrey, A. Petrukhin, D. Pitzl, A. Raspereza, P.M. Ribeiro Cipriano, C. Riedl, E. Ron, M. Rosin, J. SalfeldNebgen, R. Schmidt ${ }^{18}$, T. Schoerner-Sadenius, N. Sen, A. Spiridonov, M. Stein, R. Walsh, C. Wissing

\section{University of Hamburg, Hamburg, Germany}

V. Blobel, H. Enderle, J. Erfle, U. Gebbert, M. Görner, M. Gosselink, J. Haller,

T. Hermanns, R.S. Höing, K. Kaschube, G. Kaussen, H. Kirschenmann, R. Klanner,

J. Lange, T. Peiffer, N. Pietsch, D. Rathjens, C. Sander, H. Schettler, P. Schleper, E. Schlieckau, A. Schmidt, M. Schröder, T. Schum, M. Seidel, J. Sibille ${ }^{19}$, V. Sola, H. Stadie, G. Steinbrück, J. Thomsen, L. Vanelderen

\section{Institut für Experimentelle Kernphysik, Karlsruhe, Germany}

C. Barth, C. Baus, J. Berger, C. Böser, T. Chwalek, W. De Boer, A. Descroix, A. Dierlamm, M. Feindt, M. Guthoff ${ }^{2}$, C. Hackstein, F. Hartmann ${ }^{2}$, T. Hauth ${ }^{2}$, M. Heinrich, H. Held, K.H. Hoffmann, U. Husemann, I. Katkov ${ }^{17}$, J.R. Komaragiri, P. Lobelle Pardo, D. Martschei, S. Mueller, Th. Müller, M. Niegel, A. Nürnberg, O. Oberst, A. Oehler, J. Ott, G. Quast, K. Rabbertz, F. Ratnikov, N. Ratnikova, S. Röcker, F.-P. Schilling, G. Schott, H.J. Simonis, F.M. Stober, D. Troendle, R. Ulrich, J. Wagner-Kuhr, S. Wayand, T. Weiler, M. Zeise

Institute of Nuclear Physics "Demokritos", Aghia Paraskevi, Greece

G. Anagnostou, G. Daskalakis, T. Geralis, S. Kesisoglou, A. Kyriakis, D. Loukas, I. Manolakos, A. Markou, C. Markou, E. Ntomari

\section{University of Athens, Athens, Greece}

L. Gouskos, T.J. Mertzimekis, A. Panagiotou, N. Saoulidou

University of Ioánnina, Ioánnina, Greece

I. Evangelou, C. Foudas, P. Kokkas, N. Manthos, I. Papadopoulos

KFKI Research Institute for Particle and Nuclear Physics, Budapest, Hungary G. Bencze, C. Hajdu, P. Hidas, D. Horvath ${ }^{20}$, F. Sikler, V. Veszpremi, G. Vesztergombi ${ }^{21}$, A.J. Zsigmond 
Institute of Nuclear Research ATOMKI, Debrecen, Hungary

N. Beni, S. Czellar, J. Molnar, J. Palinkas, Z. Szillasi

University of Debrecen, Debrecen, Hungary

J. Karancsi, P. Raics, Z.L. Trocsanyi, B. Ujvari

Panjab University, Chandigarh, India

S.B. Beri, V. Bhatnagar, N. Dhingra, R. Gupta, M. Kaur, M.Z. Mehta, M. Mittal, N. Nishu, L.K. Saini, A. Sharma, J.B. Singh

University of Delhi, Delhi, India

Ashok Kumar, Arun Kumar, S. Ahuja, A. Bhardwaj, B.C. Choudhary, S. Malhotra, M. Naimuddin, K. Ranjan, P. Saxena, V. Sharma, R.K. Shivpuri

\section{Saha Institute of Nuclear Physics, Kolkata, India}

S. Banerjee, S. Bhattacharya, K. Chatterjee, S. Dutta, B. Gomber, Sa. Jain, Sh. Jain, R. Khurana, A. Modak, S. Mukherjee, D. Roy, S. Sarkar, M. Sharan

Bhabha Atomic Research Centre, Mumbai, India

A. Abdulsalam, D. Dutta, S. Kailas, V. Kumar, A.K. Mohanty², L.M. Pant, P. Shukla

Tata Institute of Fundamental Research - EHEP, Mumbai, India

T. Aziz, R.M. Chatterjee, S. Ganguly, M. Guchait ${ }^{22}$, A. Gurtu ${ }^{23}$, M. Maity ${ }^{24}$, G. Majumder, K. Mazumdar, G.B. Mohanty, B. Parida, K. Sudhakar, N. Wickramage

Tata Institute of Fundamental Research - HECR, Mumbai, India

S. Banerjee, S. Dugad

Institute for Research in Fundamental Sciences (IPM), Tehran, Iran

H. Arfaei ${ }^{25}$, H. Bakhshiansohi, S.M. Etesami ${ }^{26}$, A. Fahim ${ }^{25}$, M. Hashemi ${ }^{27}$, H. Hesari,

A. Jafari, M. Khakzad, M. Mohammadi Najafabadi, S. Paktinat Mehdiabadi, B. Safarzadeh ${ }^{28}$, M. Zeinali

INFN Sezione di Bari ${ }^{a}$, Università di Bari ${ }^{b}$, Politecnico di Bari ${ }^{c}$, Bari, Italy M. Abbrescia $^{a, b}$, L. Barbone ${ }^{a, b}$, C. Calabria ${ }^{a, b, 2}$, S.S. Chhibra ${ }^{a, b}$, A. Colaleo ${ }^{a}$, D. Creanza ${ }^{a, c}$, N. De Filippis ${ }^{a, c, 2}$, M. De Palma ${ }^{a, b}$, L. Fiore ${ }^{a}$, G. Iaselli ${ }^{a, c}$, G. Maggi ${ }^{a, c}$, M. Maggi ${ }^{a}$, B. Marangelli ${ }^{a, b}$, S. $\mathrm{My}^{a, c}$, S. $\mathrm{Nuzzo}^{a, b}$, N. Pacifico ${ }^{a}$, A. Pompili ${ }^{a, b}$, G. Pugliese ${ }^{a, c}$, G. Selvaggi ${ }^{a, b}$, L. Silvestris ${ }^{a}$, G. Singh ${ }^{a, b}$, R. Venditti ${ }^{a, b}$, P. Verwilligen ${ }^{a}$, G. Zito ${ }^{a}$

\section{INFN Sezione di Bologna ${ }^{a}$, Università di Bologna ${ }^{b}$, Bologna, Italy}

G. Abbiendi ${ }^{a}$, A.C. Benvenuti ${ }^{a}$, D. Bonacorsi ${ }^{a}, b$, S. Braibant-Giacomelli ${ }^{a, b}$, L. Brigliadori ${ }^{a, b}$, P. Capiluppi ${ }^{a, b}$, A. Castro ${ }^{a, b}$, F.R. Cavallo ${ }^{a}$, M. Cuffiani ${ }^{a, b}$, G.M. Dallavalle ${ }^{a}$, F. Fabbri ${ }^{a}$, A. Fanfani ${ }^{a}, b$, D. Fasanella ${ }^{a, b}$, P. Giacomelli ${ }^{a}$, C. Grandi ${ }^{a}$, L. Guiducci ${ }^{a, b}$, S. Marcellini ${ }^{a}$, G. Masetti ${ }^{a}$, M. Meneghelli ${ }^{a, b, 2}$, A. Montanari ${ }^{a}$,

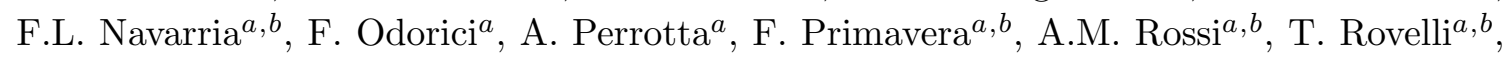
G.P. Siroli ${ }^{a, b}$, N. Tosi, R. Travaglini ${ }^{a, b}$ 
INFN Sezione di Catania ${ }^{a}$, Università di Catania ${ }^{b}$, Catania, Italy

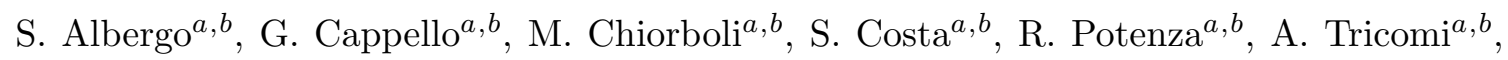

C. Tuve ${ }^{a, b}$

INFN Sezione di Firenze ${ }^{a}$, Università di Firenze ${ }^{b}$, Firenze, Italy

G. Barbagli ${ }^{a}$, V. Ciulli ${ }^{a, b}$, C. Civinini ${ }^{a}$, R. D’Alessandro ${ }^{a, b}$, E. Focardi ${ }^{a}, b$, S. Frosali ${ }^{a, b}$,

E. Gallo ${ }^{a}$, S. Gonzi ${ }^{a}, b$, M. Meschini ${ }^{a}$, S. Paoletti ${ }^{a}$, G. Sguazzoni $^{a}$, A. Tropiano $^{a, b}$

INFN Laboratori Nazionali di Frascati, Frascati, Italy

L. Benussi, S. Bianco, S. Colafranceschi ${ }^{29}$, F. Fabbri, D. Piccolo

INFN Sezione di Genova ${ }^{a}$, Università di Genova ${ }^{b}$, Genova, Italy

P. Fabbricatore ${ }^{a}$, R. Musenich ${ }^{a}$, S. Tosi ${ }^{a, b}$

INFN Sezione di Milano-Bicocca ${ }^{a}$, Università di Milano-Bicocca ${ }^{b}$, Milano, Italy

A. Benaglia $^{a}$, F. De Guio ${ }^{a, b}$, L. Di Matteo ${ }^{a, b, 2}$, S. Fiorendi $^{a}, b$, S. Gennai ${ }^{a, 2}$, A. Ghezzi $^{a, b}$, M.T. Lucchini ${ }^{2}$, S. Malvezzi ${ }^{a}$, R.A. Manzoni ${ }^{a, b}$, A. Martelli ${ }^{a}, b$ A. Massironi ${ }^{a, b}$,

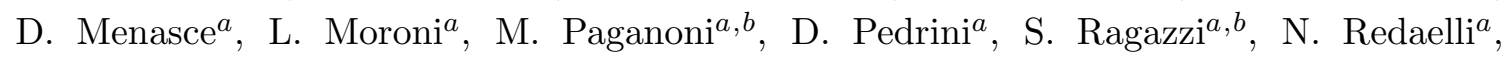
T. Tabarelli de Fatis ${ }^{a, b}$

INFN Sezione di Napoli ${ }^{a}$, Università di Napoli "Federico II" ${ }^{b}$, Napoli, Italy S. Buontempo ${ }^{a}$, N. Cavallo ${ }^{a, 30}$, A. De Cosa $a^{a, b, 2}$, O. Dogangun ${ }^{a, b}$, F. Fabozzi ${ }^{a, 30}$, A.O.M. Iorio ${ }^{a, b}$, L. Lista $^{a}$, S. Meola ${ }^{a, 31}$, M. Merola ${ }^{a}$, P. Paolucci ${ }^{a, 2}$

INFN Sezione di Padova ${ }^{a}$, Università di Padova ${ }^{b}$, Università di Trento (Trento) ${ }^{c}$, Padova, Italy

P. Azzi ${ }^{a}$, N. Bacchetta ${ }^{a, 2}$, P. Bellan ${ }^{a, b}$, D. Bisello ${ }^{a, b}$, A. Branca ${ }^{a, b, 2}$, R. Carlin $^{a, b}$, P. Checchia ${ }^{a}$, T. Dorigo ${ }^{a}$, U. Dosselli $^{a}$, F. Gasparini ${ }^{a, b}$, U. Gasparini $^{a, b}$, A. Gozzelino $^{a}$, K. Kanishchev ${ }^{a, c}$, S. Lacaprara ${ }^{a}$, I. Lazzizzera ${ }^{a, c}$, M. Margoni $^{a, b}$, A.T. Meneguzzo ${ }^{a, b}$, M. Nespolo ${ }^{a, 2}$, J. Pazzini ${ }^{a, b}$, P. Ronchese ${ }^{a, b}$, F. Simonetto ${ }^{a, b}$, E. Torassa $^{a}$, S. Vanini ${ }^{a, b}$, P. Zotto ${ }^{a, b}$, G. Zumerle ${ }^{a, b}$

INFN Sezione di Pavia ${ }^{a}$, Università di Pavia ${ }^{b}$, Pavia, Italy

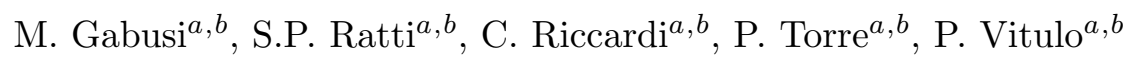

INFN Sezione di Perugia ${ }^{a}$, Università di Perugia ${ }^{b}$, Perugia, Italy

M. Biasini ${ }^{a}, b$, G.M. Bilei ${ }^{a}$, L. Fanò ${ }^{a, b}$, P. Lariccia ${ }^{a, b}$, G. Mantovani ${ }^{a}, b$, M. Menichelli ${ }^{a}$, A. Nappi ${ }^{a, b \dagger}$, F. Romeo ${ }^{a, b}$, A. Saha ${ }^{a}$, A. Santocchia ${ }^{a, b}$, A. Spiezia ${ }^{a, b}$, S. Taroni ${ }^{a, b}$

INFN Sezione di Pisa ${ }^{a}$, Università di Pisa ${ }^{b}$, Scuola Normale Superiore di Pisa ${ }^{c}$, Pisa, Italy

P. Azzurri ${ }^{a, c}$, G. Bagliesi ${ }^{a}$, J. Bernardini ${ }^{a}$, T. Boccali ${ }^{a}$, G. Broccolo ${ }^{a, c}$, R. Castaldi $^{a}$, R.T. D'Agnolo ${ }^{a, c, 2}$, R. Dell'Orso ${ }^{a}$, F. Fiori ${ }^{a, b, 2}$, L. Foà ${ }^{a, c}$, A. Giassi ${ }^{a}$, A. Kraan ${ }^{a}$, F. $\operatorname{Ligabue}^{a, c}$, T. $\operatorname{Lomtadze}^{a}$, L. Martini ${ }^{a, 32}$, A. Messineo ${ }^{a, b}$, F. Palla ${ }^{a}$, A. Rizzi ${ }^{a, b}$,

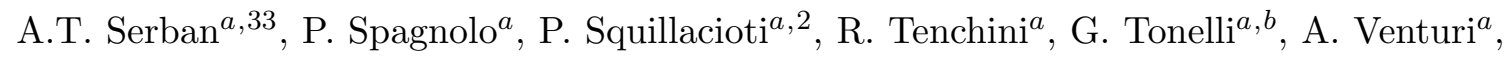
P.G. Verdini ${ }^{a}$ 
INFN Sezione di Roma ${ }^{a}$, Università di Roma ${ }^{b}$, Roma, Italy

L. Barone ${ }^{a, b}$, F. Cavallari ${ }^{a}$, D. Del Re $\mathrm{Re}^{a, b}$, M. Diemoz ${ }^{a}$, C. Fanellia ${ }^{a, b}$, M. Grassi ${ }^{a, b, 2}$, E. Longo ${ }^{a, b}$, P. Meridiani ${ }^{a, 2}$, F. Micheli ${ }^{a, b}$, S. Nourbakhsh ${ }^{a, b}$, G. Organtini ${ }^{a, b}$, R. Paramatti ${ }^{a}$, S. Rahatlou ${ }^{a, b}$, L. Soffi ${ }^{a, b}$

INFN Sezione di Torino ${ }^{a}$, Università di Torino ${ }^{b}$, Università del Piemonte Orientale (Novara) ${ }^{c}$, Torino, Italy

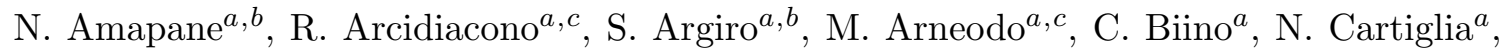

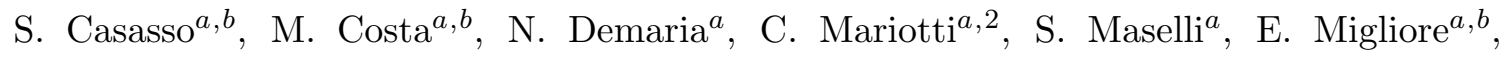
V. Monaco ${ }^{a, b}$, M. Musich ${ }^{a, 2}$, M.M. Obertino ${ }^{a, c}$, N. Pastrone ${ }^{a}$, M. Pelliccioni ${ }^{a}$,

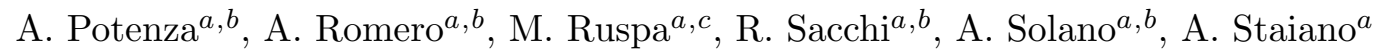

INFN Sezione di Trieste ${ }^{a}$, Università di Trieste ${ }^{b}$, Trieste, Italy

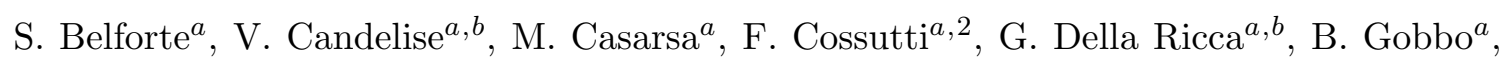
M. Marone ${ }^{a, b, 2}$, D. Montanino ${ }^{a, b}$, A. Penzo $^{a}$, A. Schizzi ${ }^{a, b}$

Kangwon National University, Chunchon, Korea

T.Y. Kim, S.K. Nam

Kyungpook National University, Daegu, Korea

S. Chang, D.H. Kim, G.N. Kim, D.J. Kong, H. Park, D.C. Son

Chonnam National University, Institute for Universe and Elementary Particles, Kwangju, Korea

J.Y. Kim, Zero J. Kim, S. Song

Korea University, Seoul, Korea

S. Choi, D. Gyun, B. Hong, M. Jo, H. Kim, T.J. Kim, K.S. Lee, D.H. Moon, S.K. Park, Y. Roh

University of Seoul, Seoul, Korea

M. Choi, J.H. Kim, C. Park, I.C. Park, S. Park, G. Ryu

Sungkyunkwan University, Suwon, Korea

Y. Choi, Y.K. Choi, J. Goh, M.S. Kim, E. Kwon, B. Lee, J. Lee, S. Lee, H. Seo, I. Yu

Vilnius University, Vilnius, Lithuania

M.J. Bilinskas, I. Grigelionis, M. Janulis, A. Juodagalvis

Centro de Investigacion y de Estudios Avanzados del IPN, Mexico City, Mexico

H. Castilla-Valdez, E. De La Cruz-Burelo, I. Heredia-de La Cruz, R. Lopez-Fernandez, J. Martínez-Ortega, A. Sanchez-Hernandez, L.M. Villasenor-Cendejas

Universidad Iberoamericana, Mexico City, Mexico

S. Carrillo Moreno, F. Vazquez Valencia

Benemerita Universidad Autonoma de Puebla, Puebla, Mexico H.A. Salazar Ibarguen 
Universidad Autónoma de San Luis Potosí, San Luis Potosí, Mexico

E. Casimiro Linares, A. Morelos Pineda, M.A. Reyes-Santos

University of Auckland, Auckland, New Zealand

D. Krofcheck

University of Canterbury, Christchurch, New Zealand

A.J. Bell, P.H. Butler, R. Doesburg, S. Reucroft, H. Silverwood

National Centre for Physics, Quaid-I-Azam University, Islamabad, Pakistan

M. Ahmad, M.I. Asghar, J. Butt, H.R. Hoorani, S. Khalid, W.A. Khan, T. Khurshid, S. Qazi, M.A. Shah, M. Shoaib

National Centre for Nuclear Research, Swierk, Poland

H. Bialkowska, B. Boimska, T. Frueboes, M. Górski, M. Kazana, K. Nawrocki,

K. Romanowska-Rybinska, M. Szleper, G. Wrochna, P. Zalewski

Institute of Experimental Physics, Faculty of Physics, University of Warsaw, Warsaw, Poland

G. Brona, K. Bunkowski, M. Cwiok, W. Dominik, K. Doroba, A. Kalinowski, M. Konecki, J. Krolikowski, M. Misiura, W. Wolszczak

Laboratório de Instrumentação e Física Experimental de Partículas, Lisboa, Portugal

N. Almeida, P. Bargassa, A. David, P. Faccioli, P.G. Ferreira Parracho, M. Gallinaro, J. Seixas, J. Varela, P. Vischia

Joint Institute for Nuclear Research, Dubna, Russia

I. Belotelov, P. Bunin, M. Gavrilenko, I. Golutvin, I. Gorbunov, A. Kamenev, V. Karjavin, G. Kozlov, A. Lanev, A. Malakhov, P. Moisenz, V. Palichik, V. Perelygin, S. Shmatov, V. Smirnov, A. Volodko, A. Zarubin

Petersburg Nuclear Physics Institute, Gatchina (St. Petersburg), Russia

S. Evstyukhin, V. Golovtsov, Y. Ivanov, V. Kim, P. Levchenko, V. Murzin, V. Oreshkin, I. Smirnov, V. Sulimov, L. Uvarov, S. Vavilov, A. Vorobyev, An. Vorobyev

Institute for Nuclear Research, Moscow, Russia

Yu. Andreev, A. Dermenev, S. Gninenko, N. Golubev, M. Kirsanov, N. Krasnikov, V. Matveev, A. Pashenkov, D. Tlisov, A. Toropin

Institute for Theoretical and Experimental Physics, Moscow, Russia

V. Epshteyn, M. Erofeeva, V. Gavrilov, M. Kossov, N. Lychkovskaya, V. Popov, G. Safronov, S. Semenov, I. Shreyber, V. Stolin, E. Vlasov, A. Zhokin

Moscow State University, Moscow, Russia

A. Belyaev, E. Boos, M. Dubinin ${ }^{6}$, L. Dudko, A. Ershov, A. Gribushin, V. Klyukhin, O. Kodolova, I. Lokhtin, A. Markina, S. Obraztsov, M. Perfilov, S. Petrushanko, A. Popov, L. Sarycheva ${ }^{\dagger}$, V. Savrin, A. Snigirev 
P.N. Lebedev Physical Institute, Moscow, Russia

V. Andreev, M. Azarkin, I. Dremin, M. Kirakosyan, A. Leonidov, G. Mesyats, S.V. Rusakov, A. Vinogradov

State Research Center of Russian Federation, Institute for High Energy Physics, Protvino, Russia

I. Azhgirey, I. Bayshev, S. Bitioukov, V. Grishin ${ }^{2}$, V. Kachanov, D. Konstantinov,

V. Krychkine, V. Petrov, R. Ryutin, A. Sobol, L. Tourtchanovitch, S. Troshin, N. Tyurin,

A. Uzunian, A. Volkov

University of Belgrade, Faculty of Physics and Vinca Institute of Nuclear Sciences, Belgrade, Serbia

P. Adzic ${ }^{34}$, M. Djordjevic, M. Ekmedzic, D. Krpic ${ }^{34}$, J. Milosevic

Centro de Investigaciones Energéticas Medioambientales y Tecnológicas (CIEMAT), Madrid, Spain

M. Aguilar-Benitez, J. Alcaraz Maestre, P. Arce, C. Battilana, E. Calvo, M. Cerrada,

M. Chamizo Llatas, N. Colino, B. De La Cruz, A. Delgado Peris, D. Domínguez Vázquez,

C. Fernandez Bedoya, J.P. Fernández Ramos, A. Ferrando, J. Flix, M.C. Fouz, P. GarciaAbia, O. Gonzalez Lopez, S. Goy Lopez, J.M. Hernandez, M.I. Josa, G. Merino, J. Puerta Pelayo, A. Quintario Olmeda, I. Redondo, L. Romero, J. Santaolalla, M.S. Soares, C. Willmott

Universidad Autónoma de Madrid, Madrid, Spain

C. Albajar, G. Codispoti, J.F. de Trocóniz

Universidad de Oviedo, Oviedo, Spain

H. Brun, J. Cuevas, J. Fernandez Menendez, S. Folgueras, I. Gonzalez Caballero, L. Lloret Iglesias, J. Piedra Gomez

Instituto de Física de Cantabria (IFCA), CSIC-Universidad de Cantabria, Santander, Spain

J.A. Brochero Cifuentes, I.J. Cabrillo, A. Calderon, S.H. Chuang, J. Duarte Campderros, M. Felcini ${ }^{35}$, M. Fernandez, G. Gomez, J. Gonzalez Sanchez, A. Graziano, C. Jorda, A. Lopez Virto, J. Marco, R. Marco, C. Martinez Rivero, F. Matorras, F.J. Munoz Sanchez, T. Rodrigo, A.Y. Rodríguez-Marrero, A. Ruiz-Jimeno, L. Scodellaro, I. Vila, R. Vilar Cortabitarte

\section{CERN, European Organization for Nuclear Research, Geneva, Switzerland}

D. Abbaneo, E. Auffray, G. Auzinger, M. Bachtis, P. Baillon, A.H. Ball, D. Barney, J.F. Benitez, C. Bernet ${ }^{7}$, G. Bianchi, P. Bloch, A. Bocci, A. Bonato, C. Botta, H. Breuker, T. Camporesi, G. Cerminara, T. Christiansen, J.A. Coarasa Perez, D. D'Enterria, A. Dabrowski, A. De Roeck, S. De Visscher, S. Di Guida, M. Dobson, N. DupontSagorin, A. Elliott-Peisert, B. Frisch, W. Funk, G. Georgiou, M. Giffels, D. Gigi, K. Gill, D. Giordano, M. Girone, M. Giunta, F. Glege, R. Gomez-Reino Garrido, P. Govoni, S. Gowdy, R. Guida, J. Hammer, M. Hansen, P. Harris, C. Hartl, J. Harvey, B. Hegner, A. Hinzmann, V. Innocente, P. Janot, K. Kaadze, E. Karavakis, K. Kousouris, P. Lecoq, 
Y.-J. Lee, P. Lenzi, C. Lourenço, N. Magini, T. Mäki, M. Malberti, L. Malgeri, M. Mannelli, L. Masetti, F. Meijers, S. Mersi, E. Meschi, R. Moser, M. Mulders, P. Musella, E. Nesvold, L. Orsini, E. Palencia Cortezon, E. Perez, L. Perrozzi, A. Petrilli, A. Pfeiffer, M. Pierini, M. Pimiä, D. Piparo, G. Polese, L. Quertenmont, A. Racz, W. Reece, J. Rodrigues Antunes, G. Rolandi ${ }^{36}$, C. Rovelli ${ }^{37}$, M. Rovere, H. Sakulin, F. Santanastasio, C. Schäfer, C. Schwick, I. Segoni, S. Sekmen, A. Sharma, P. Siegrist, P. Silva, M. Simon, P. Sphicas ${ }^{38}$, D. Spiga, A. Tsirou, G.I. Veres ${ }^{21}$, J.R. Vlimant, H.K. Wöhri, S.D. Worm ${ }^{39}$, W.D. Zeuner

\section{Paul Scherrer Institut, Villigen, Switzerland}

W. Bertl, K. Deiters, W. Erdmann, K. Gabathuler, R. Horisberger, Q. Ingram, H.C. Kaestli, S. König, D. Kotlinski, U. Langenegger, F. Meier, D. Renker, T. Rohe

Institute for Particle Physics, ETH Zurich, Zurich, Switzerland

F. Bachmair, L. Bäni, P. Bortignon, M.A. Buchmann, B. Casal, N. Chanon, A. Deisher, G. Dissertori, M. Dittmar, M. Donegà, M. Dünser, P. Eller, J. Eugster, K. Freudenreich, C. Grab, D. Hits, P. Lecomte, W. Lustermann, A.C. Marini, P. Martinez Ruiz del Arbol, N. Mohr, F. Moortgat, C. Nägeli40 ${ }^{40}$ P. Nef, F. Nessi-Tedaldi, F. Pandolfi, L. Pape, F. Pauss, M. Peruzzi, F.J. Ronga, M. Rossini, L. Sala, A.K. Sanchez, A. Starodumov ${ }^{41}$, B. Stieger, M. Takahashi, L. Tauscher ${ }^{\dagger}$, A. Thea, K. Theofilatos, D. Treille, C. Urscheler, R. Wallny, H.A. Weber, L. Wehrli

\section{Universität Zürich, Zurich, Switzerland}

C. Amsler ${ }^{42}$, V. Chiochia, C. Favaro, M. Ivova Rikova, B. Kilminster, B. Millan Mejias, P. Otiougova, P. Robmann, H. Snoek, S. Tupputi, M. Verzetti

\section{National Central University, Chung-Li, Taiwan}

Y.H. Chang, K.H. Chen, C. Ferro, C.M. Kuo, S.W. Li, W. Lin, Y.J. Lu, A.P. Singh, R. Volpe, S.S. Yu

\section{National Taiwan University (NTU), Taipei, Taiwan}

P. Bartalini, P. Chang, Y.H. Chang, Y.W. Chang, Y. Chao, K.F. Chen, C. Dietz, U. Grundler, W.-S. Hou, Y. Hsiung, K.Y. Kao, Y.J. Lei, R.-S. Lu, D. Majumder, E. Petrakou, X. Shi, J.G. Shiu, Y.M. Tzeng, X. Wan, M. Wang

\section{Chulalongkorn University, Bangkok, Thailand}

B. Asavapibhop, E. Simili, N. Srimanobhas, N. Suwonjandee

\section{Cukurova University, Adana, Turkey}

A. Adiguzel, M.N. Bakirci ${ }^{43}$, S. $\mathrm{Cerci}^{44}$, C. Dozen, I. Dumanoglu, E. Eskut, S. Girgis, G. Gokbulut, E. Gurpinar, I. Hos, E.E. Kangal, T. Karaman, G. Karapinar ${ }^{45}$, A. Kayis Topaksu, G. Onengut, K. Ozdemir, S. Ozturk ${ }^{46}$, A. Polatoz, K. Sogut ${ }^{47}$, D. Sunar Cerci ${ }^{44}$, B. Tali ${ }^{44}$, H. Topakli ${ }^{43}$, L.N. Vergili, M. Vergili

Middle East Technical University, Physics Department, Ankara, Turkey I.V. Akin, T. Aliev, B. Bilin, S. Bilmis, M. Deniz, H. Gamsizkan, A.M. Guler, K. Ocalan, A. Ozpineci, M. Serin, R. Sever, U.E. Surat, M. Yalvac, E. Yildirim, M. Zeyrek 
Bogazici University, Istanbul, Turkey

E. Gülmez, B. Isildak ${ }^{48}$, M. Kaya ${ }^{49}$, O. Kaya ${ }^{49}$, S. Ozkorucuklu ${ }^{50}$, N. Sonmez ${ }^{51}$

Istanbul Technical University, Istanbul, Turkey

H. Bahtiyar ${ }^{52}$, E. Barlas, K. Cankocak, Y.O. Günaydin ${ }^{53}$, F.I. Vardarlı, M. Yücel

National Scientific Center, Kharkov Institute of Physics and Technology, Kharkov, Ukraine

L. Levchuk

University of Bristol, Bristol, United Kingdom

J.J. Brooke, E. Clement, D. Cussans, H. Flacher, R. Frazier, J. Goldstein, M. Grimes, G.P. Heath, H.F. Heath, L. Kreczko, S. Metson, D.M. Newbold ${ }^{39}$, K. Nirunpong, A. Poll, S. Senkin, V.J. Smith, T. Williams

\section{Rutherford Appleton Laboratory, Didcot, United Kingdom}

L. Basso ${ }^{54}$, K.W. Bell, A. Belyaev ${ }^{54}$, C. Brew, R.M. Brown, D.J.A. Cockerill, J.A. Coughlan, K. Harder, S. Harper, J. Jackson, B.W. Kennedy, E. Olaiya, D. Petyt, B.C. RadburnSmith, C.H. Shepherd-Themistocleous, I.R. Tomalin, W.J. Womersley

\section{Imperial College, London, United Kingdom}

R. Bainbridge, G. Ball, R. Beuselinck, O. Buchmuller, D. Colling, N. Cripps, M. Cutajar, P. Dauncey, G. Davies, M. Della Negra, W. Ferguson, J. Fulcher, D. Futyan, A. Gilbert, A. Guneratne Bryer, G. Hall, Z. Hatherell, J. Hays, G. Iles, M. Jarvis, G. Karapostoli, M. Kenzie, L. Lyons, A.-M. Magnan, J. Marrouche, B. Mathias, R. Nandi, J. Nash, A. Nikitenko ${ }^{41}$, J. Pela, M. Pesaresi, K. Petridis, M. Pioppi ${ }^{55}$, D.M. Raymond, S. Rogerson, A. Rose, C. Seez, P. Sharp ${ }^{\dagger}$, A. Sparrow, M. Stoye, A. Tapper, M. Vazquez Acosta, T. Virdee, S. Wakefield, N. Wardle, T. Whyntie

\section{Brunel University, Uxbridge, United Kingdom}

M. Chadwick, J.E. Cole, P.R. Hobson, A. Khan, P. Kyberd, D. Leggat, D. Leslie, W. Martin, I.D. Reid, P. Symonds, L. Teodorescu, M. Turner

Baylor University, Waco, USA

K. Hatakeyama, H. Liu, T. Scarborough

The University of Alabama, Tuscaloosa, USA

O. Charaf, S.I. Cooper, C. Henderson, P. Rumerio

\section{Boston University, Boston, USA}

A. Avetisyan, T. Bose, C. Fantasia, A. Heister, J. St. John, P. Lawson, D. Lazic, J. Rohlf, D. Sperka, L. Sulak

\section{Brown University, Providence, USA}

J. Alimena, S. Bhattacharya, G. Christopher, D. Cutts, Z. Demiragli, A. Ferapontov, A. Garabedian, U. Heintz, S. Jabeen, G. Kukartsev, E. Laird, G. Landsberg, M. Luk, M. Narain, M. Segala, T. Sinthuprasith, T. Speer 


\section{University of California, Davis, Davis, USA}

R. Breedon, G. Breto, M. Calderon De La Barca Sanchez, M. Caulfield, S. Chauhan, M. Chertok, J. Conway, R. Conway, P.T. Cox, J. Dolen, R. Erbacher, M. Gardner, R. Houtz, W. Ko, A. Kopecky, R. Lander, O. Mall, T. Miceli, R. Nelson, D. Pellett, F. Ricci-Tam, B. Rutherford, M. Searle, J. Smith, M. Squires, M. Tripathi, R. Vasquez Sierra, R. Yohay

\section{University of California, Los Angeles, Los Angeles, USA}

V. Andreev, D. Cline, R. Cousins, J. Duris, S. Erhan, P. Everaerts, C. Farrell, J. Hauser, M. Ignatenko, C. Jarvis, G. Rakness, P. Schlein ${ }^{\dagger}$, P. Traczyk, V. Valuev, M. Weber

University of California, Riverside, Riverside, USA

J. Babb, R. Clare, M.E. Dinardo, J. Ellison, J.W. Gary, F. Giordano, G. Hanson, H. Liu, O.R. Long, A. Luthra, H. Nguyen, S. Paramesvaran, J. Sturdy, S. Sumowidagdo, R. Wilken, S. Wimpenny

\section{University of California, San Diego, La Jolla, USA}

W. Andrews, J.G. Branson, G.B. Cerati, S. Cittolin, D. Evans, A. Holzner, R. Kelley, M. Lebourgeois, J. Letts, I. Macneill, B. Mangano, S. Padhi, C. Palmer, G. Petrucciani, M. Pieri, M. Sani, V. Sharma, S. Simon, E. Sudano, M. Tadel, Y. Tu, A. Vartak, S. Wasserbaech ${ }^{56}$, F. Würthwein, A. Yagil, J. Yoo

\section{University of California, Santa Barbara, Santa Barbara, USA}

D. Barge, R. Bellan, C. Campagnari, M. D’Alfonso, T. Danielson, K. Flowers, P. Geffert, C. George, F. Golf, J. Incandela, C. Justus, P. Kalavase, D. Kovalskyi, V. Krutelyov, S. Lowette, R. Magaña Villalba, N. Mccoll, V. Pavlunin, J. Ribnik, J. Richman, R. Rossin, D. Stuart, W. To, C. West

\section{California Institute of Technology, Pasadena, USA}

A. Apresyan, A. Bornheim, Y. Chen, E. Di Marco, J. Duarte, M. Gataullin, Y. Ma, A. Mott, H.B. Newman, C. Rogan, M. Spiropulu, V. Timciuc, J. Veverka, R. Wilkinson, S. Xie, Y. Yang, R.Y. Zhu

\section{Carnegie Mellon University, Pittsburgh, USA}

V. Azzolini, A. Calamba, R. Carroll, T. Ferguson, Y. Iiyama, D.W. Jang, Y.F. Liu, M. Paulini, H. Vogel, I. Vorobiev

\section{University of Colorado at Boulder, Boulder, USA}

J.P. Cumalat, B.R. Drell, W.T. Ford, A. Gaz, E. Luiggi Lopez, J.G. Smith, K. Stenson, K.A. Ulmer, S.R. Wagner

\section{Cornell University, Ithaca, USA}

J. Alexander, A. Chatterjee, N. Eggert, L.K. Gibbons, B. Heltsley, W. Hopkins, A. Khukhunaishvili, B. Kreis, N. Mirman, G. Nicolas Kaufman, J.R. Patterson, A. Ryd, E. Salvati, W. Sun, W.D. Teo, J. Thom, J. Thompson, J. Tucker, Y. Weng, L. Winstrom, P. Wittich Fairfield University, Fairfield, USA

D. Winn 


\section{Fermi National Accelerator Laboratory, Batavia, USA}

S. Abdullin, M. Albrow, J. Anderson, L.A.T. Bauerdick, A. Beretvas, J. Berryhill, P.C. Bhat, K. Burkett, J.N. Butler, V. Chetluru, H.W.K. Cheung, F. Chlebana, V.D. Elvira, I. Fisk, J. Freeman, Y. Gao, D. Green, O. Gutsche, J. Hanlon, R.M. Harris, J. Hirschauer, B. Hooberman, S. Jindariani, M. Johnson, U. Joshi, B. Klima, S. Kunori, S. Kwan, C. Leonidopoulos ${ }^{57}$, J. Linacre, D. Lincoln, R. Lipton, J. Lykken, K. Maeshima, J.M. Marraffino, V.I. Martinez Outschoorn, S. Maruyama, D. Mason, P. McBride, K. Mishra, S. Mrenna, Y. Musienko ${ }^{58}$, C. Newman-Holmes, V. O'Dell, O. Prokofyev, E. Sexton-Kennedy, S. Sharma, W.J. Spalding, L. Spiegel, L. Taylor, S. Tkaczyk, N.V. Tran, L. Uplegger, E.W. Vaandering, R. Vidal, J. Whitmore, W. Wu, F. Yang, J.C. Yun

\section{University of Florida, Gainesville, USA}

D. Acosta, P. Avery, D. Bourilkov, M. Chen, T. Cheng, S. Das, M. De Gruttola, G.P. Di Giovanni, D. Dobur, A. Drozdetskiy, R.D. Field, M. Fisher, Y. Fu, I.K. Furic, J. Gartner, J. Hugon, B. Kim, J. Konigsberg, A. Korytov, A. Kropivnitskaya, T. Kypreos, J.F. Low, K. Matchev, P. Milenovic ${ }^{59}$, G. Mitselmakher, L. Muniz, M. Park, R. Remington, A. Rinkevicius, P. Sellers, N. Skhirtladze, M. Snowball, J. Yelton, M. Zakaria

\section{Florida International University, Miami, USA}

V. Gaultney, S. Hewamanage, L.M. Lebolo, S. Linn, P. Markowitz, G. Martinez, J.L. Rodriguez

\section{Florida State University, Tallahassee, USA}

T. Adams, A. Askew, J. Bochenek, J. Chen, B. Diamond, S.V. Gleyzer, J. Haas,

S. Hagopian, V. Hagopian, M. Jenkins, K.F. Johnson, H. Prosper, V. Veeraraghavan, M. Weinberg

\section{Florida Institute of Technology, Melbourne, USA}

M.M. Baarmand, B. Dorney, M. Hohlmann, H. Kalakhety, I. Vodopiyanov, F. Yumiceva

\section{University of Illinois at Chicago (UIC), Chicago, USA}

M.R. Adams, L. Apanasevich, Y. Bai, V.E. Bazterra, R.R. Betts, I. Bucinskaite, J. Callner, R. Cavanaugh, O. Evdokimov, L. Gauthier, C.E. Gerber, D.J. Hofman, S. Khalatyan, F. Lacroix, C. O'Brien, C. Silkworth, D. Strom, P. Turner, N. Varelas

\section{The University of Iowa, Iowa City, USA}

U. Akgun, E.A. Albayrak, B. Bilki ${ }^{60}$, W. Clarida, K. Dilsiz, F. Duru, S. Griffiths, J.P. Merlo, H. Mermerkaya ${ }^{61}$, A. Mestvirishvili, A. Moeller, J. Nachtman, C.R. Newsom, E. Norbeck, H. Ogul, Y. Onel, F. Ozok ${ }^{52}$, S. Sen, P. Tan, E. Tiras, J. Wetzel, T. Yetkin, K. Yi

\section{Johns Hopkins University, Baltimore, USA}

B.A. Barnett, B. Blumenfeld, S. Bolognesi, D. Fehling, G. Giurgiu, A.V. Gritsan, Z.J. Guo, G. Hu, P. Maksimovic, M. Swartz, A. Whitbeck 
The University of Kansas, Lawrence, USA

P. Baringer, A. Bean, G. Benelli, R.P. Kenny Iii, M. Murray, D. Noonan, S. Sanders, R. Stringer, G. Tinti, J.S. Wood

\section{Kansas State University, Manhattan, USA}

A.F. Barfuss, T. Bolton, I. Chakaberia, A. Ivanov, S. Khalil, M. Makouski, Y. Maravin, S. Shrestha, I. Svintradze

Lawrence Livermore National Laboratory, Livermore, USA

J. Gronberg, D. Lange, F. Rebassoo, D. Wright

University of Maryland, College Park, USA

A. Baden, B. Calvert, S.C. Eno, J.A. Gomez, N.J. Hadley, R.G. Kellogg, M. Kirn,

T. Kolberg, Y. Lu, M. Marionneau, A.C. Mignerey, K. Pedro, A. Peterman, A. Skuja,

J. Temple, M.B. Tonjes, S.C. Tonwar

Massachusetts Institute of Technology, Cambridge, USA

A. Apyan, G. Bauer, J. Bendavid, W. Busza, E. Butz, I.A. Cali, M. Chan, V. Dutta, G. Gomez Ceballos, M. Goncharov, Y. Kim, M. Klute, K. Krajczar ${ }^{62}$, A. Levin, P.D. Luckey, T. Ma, S. Nahn, C. Paus, D. Ralph, C. Roland, G. Roland, M. Rudolph, G.S.F. Stephans, F. Stöckli, K. Sumorok, K. Sung, D. Velicanu, E.A. Wenger, R. Wolf, B. Wyslouch, M. Yang, Y. Yilmaz, A.S. Yoon, M. Zanetti, V. Zhukova

University of Minnesota, Minneapolis, USA

B. Dahmes, A. De Benedetti, G. Franzoni, A. Gude, S.C. Kao, K. Klapoetke, Y. Kubota, J. Mans, N. Pastika, R. Rusack, M. Sasseville, A. Singovsky, N. Tambe, J. Turkewitz

University of Mississippi, Oxford, USA

L.M. Cremaldi, R. Kroeger, L. Perera, R. Rahmat, D.A. Sanders

University of Nebraska-Lincoln, Lincoln, USA

E. Avdeeva, K. Bloom, S. Bose, D.R. Claes, A. Dominguez, M. Eads, J. Keller, I. Kravchenko, J. Lazo-Flores, S. Malik, G.R. Snow

State University of New York at Buffalo, Buffalo, USA

A. Godshalk, I. Iashvili, S. Jain, A. Kharchilava, A. Kumar, S. Rappoccio, Z. Wan

Northeastern University, Boston, USA

G. Alverson, E. Barberis, D. Baumgartel, M. Chasco, J. Haley, D. Nash, T. Orimoto, D. Trocino, D. Wood, J. Zhang

Northwestern University, Evanston, USA

A. Anastassov, K.A. Hahn, A. Kubik, L. Lusito, N. Mucia, N. Odell, R.A. Ofierzynski, B. Pollack, A. Pozdnyakov, M. Schmitt, S. Stoynev, M. Velasco, S. Won

University of Notre Dame, Notre Dame, USA

D. Berry, A. Brinkerhoff, K.M. Chan, M. Hildreth, C. Jessop, D.J. Karmgard, J. Kolb, K. Lannon, W. Luo, S. Lynch, N. Marinelli, D.M. Morse, T. Pearson, M. Planer, R. Ruchti, J. Slaunwhite, N. Valls, M. Wayne, M. Wolf 


\section{The Ohio State University, Columbus, USA}

L. Antonelli, B. Bylsma, L.S. Durkin, C. Hill, R. Hughes, K. Kotov, T.Y. Ling, D. Puigh, M. Rodenburg, G. Smith, C. Vuosalo, G. Williams, B.L. Winer

Princeton University, Princeton, USA

E. Berry, P. Elmer, V. Halyo, P. Hebda, J. Hegeman, A. Hunt, P. Jindal, S.A. Koay,

D. Lopes Pegna, P. Lujan, D. Marlow, T. Medvedeva, M. Mooney, J. Olsen, P. Piroué,

X. Quan, A. Raval, H. Saka, D. Stickland, C. Tully, J.S. Werner, S.C. Zenz, A. Zuranski

University of Puerto Rico, Mayaguez, USA

E. Brownson, A. Lopez, H. Mendez, J.E. Ramirez Vargas

\section{Purdue University, West Lafayette, USA}

E. Alagoz, V.E. Barnes, D. Benedetti, G. Bolla, D. Bortoletto, M. De Mattia, A. Everett,

Z. Hu, M. Jones, O. Koybasi, M. Kress, A.T. Laasanen, N. Leonardo, V. Maroussov,

P. Merkel, D.H. Miller, N. Neumeister, I. Shipsey, D. Silvers, A. Svyatkovskiy, M. Vidal Marono, H.D. Yoo, J. Zablocki, Y. Zheng

Purdue University Calumet, Hammond, USA

S. Guragain, N. Parashar

\section{Rice University, Houston, USA}

A. Adair, B. Akgun, C. Boulahouache, K.M. Ecklund, F.J.M. Geurts, W. Li, B.P. Padley, R. Redjimi, J. Roberts, J. Zabel

University of Rochester, Rochester, USA

B. Betchart, A. Bodek, Y.S. Chung, R. Covarelli, P. de Barbaro, R. Demina, Y. Eshaq, T. Ferbel, A. Garcia-Bellido, P. Goldenzweig, J. Han, A. Harel, D.C. Miner, D. Vishnevskiy, M. Zielinski

The Rockefeller University, New York, USA

A. Bhatti, R. Ciesielski, L. Demortier, K. Goulianos, G. Lungu, S. Malik, C. Mesropian

Rutgers, the State University of New Jersey, Piscataway, USA

S. Arora, A. Barker, J.P. Chou, C. Contreras-Campana, E. Contreras-Campana, D. Duggan, D. Ferencek, Y. Gershtein, R. Gray, E. Halkiadakis, D. Hidas, A. Lath, S. Panwalkar, M. Park, R. Patel, V. Rekovic, J. Robles, K. Rose, S. Salur, S. Schnetzer, C. Seitz, S. Somalwar, R. Stone, S. Thomas, M. Walker

University of Tennessee, Knoxville, USA

G. Cerizza, M. Hollingsworth, S. Spanier, Z.C. Yang, A. York

Texas A\&M University, College Station, USA

R. Eusebi, W. Flanagan, J. Gilmore, T. Kamon ${ }^{63}$, V. Khotilovich, R. Montalvo, I. Osipenkov, Y. Pakhotin, A. Perloff, J. Roe, A. Safonov, T. Sakuma, S. Sengupta, I. Suarez, A. Tatarinov, D. Toback 


\section{Texas Tech University, Lubbock, USA}

N. Akchurin, J. Damgov, C. Dragoiu, P.R. Dudero, C. Jeong, K. Kovitanggoon, S.W. Lee, T. Libeiro, I. Volobouev

\section{Vanderbilt University, Nashville, USA}

E. Appelt, A.G. Delannoy, C. Florez, S. Greene, A. Gurrola, W. Johns, P. Kurt, C. Maguire, A. Melo, M. Sharma, P. Sheldon, B. Snook, S. Tuo, J. Velkovska

\section{University of Virginia, Charlottesville, USA}

M.W. Arenton, M. Balazs, S. Boutle, B. Cox, B. Francis, J. Goodell, R. Hirosky, A. Ledovskoy, C. Lin, C. Neu, J. Wood

\section{Wayne State University, Detroit, USA}

S. Gollapinni, R. Harr, P.E. Karchin, C. Kottachchi Kankanamge Don, P. Lamichhane, A. Sakharov

\section{University of Wisconsin, Madison, USA}

M. Anderson, D.A. Belknap, L. Borrello, D. Carlsmith, M. Cepeda, S. Dasu, E. Friis, L. Gray, K.S. Grogg, M. Grothe, R. Hall-Wilton, M. Herndon, A. Hervé, P. Klabbers, J. Klukas, A. Lanaro, C. Lazaridis, R. Loveless, A. Mohapatra, M.U. Mozer, I. Ojalvo, F. Palmonari, G.A. Pierro, I. Ross, A. Savin, W.H. Smith, J. Swanson

$\dagger$ : Deceased

1: Also at Vienna University of Technology, Vienna, Austria

2: Also at CERN, European Organization for Nuclear Research, Geneva, Switzerland

3: Also at National Institute of Chemical Physics and Biophysics, Tallinn, Estonia

4: Also at University Estadual de Campinas, Campinas, Brazil

5: Also at Universidade Federal do ABC, Santo Andre, Brazil

6: Also at California Institute of Technology, Pasadena, USA

7: Also at Laboratoire Leprince-Ringuet, Ecole Polytechnique, IN2P3-CNRS, Palaiseau, France

8: Also at Suez Canal University, Suez, Egypt

9: Also at Zewail City of Science and Technology, Zewail, Egypt

10: Also at Cairo University, Cairo, Egypt

11: Also at Fayoum University, El-Fayoum, Egypt

12: Also at British University in Egypt, Cairo, Egypt

13: Now at Ain Shams University, Cairo, Egypt

14: Also at National Centre for Nuclear Research, Swierk, Poland

15: Also at Université de Haute-Alsace, Mulhouse, France

16: Also at Joint Institute for Nuclear Research, Dubna, Russia

17: Also at Moscow State University, Moscow, Russia

18: Also at Brandenburg University of Technology, Cottbus, Germany

19: Also at The University of Kansas, Lawrence, USA

20: Also at Institute of Nuclear Research ATOMKI, Debrecen, Hungary

21: Also at Eötvös Loránd University, Budapest, Hungary

22: Also at Tata Institute of Fundamental Research - HECR, Mumbai, India

23: Now at King Abdulaziz University, Jeddah, Saudi Arabia

24: Also at University of Visva-Bharati, Santiniketan, India 
25: Also at Sharif University of Technology, Tehran, Iran

26: Also at Isfahan University of Technology, Isfahan, Iran

27: Also at Shiraz University, Shiraz, Iran

28: Also at Plasma Physics Research Center, Science and Research Branch, Islamic Azad University, Tehran, Iran

29: Also at Facoltà Ingegneria, Università di Roma, Roma, Italy

30: Also at Università della Basilicata, Potenza, Italy

31: Also at Università degli Studi Guglielmo Marconi, Roma, Italy

32: Also at Università degli Studi di Siena, Siena, Italy

33: Also at University of Bucharest, Faculty of Physics, Bucuresti-Magurele, Romania

34: Also at Faculty of Physics of University of Belgrade, Belgrade, Serbia

35: Also at University of California, Los Angeles, Los Angeles, USA

36: Also at Scuola Normale e Sezione dell'INFN, Pisa, Italy

37: Also at INFN Sezione di Roma, Roma, Italy

38: Also at University of Athens, Athens, Greece

39: Also at Rutherford Appleton Laboratory, Didcot, United Kingdom

40: Also at Paul Scherrer Institut, Villigen, Switzerland

41: Also at Institute for Theoretical and Experimental Physics, Moscow, Russia

42: Also at Albert Einstein Center for Fundamental Physics, Bern, Switzerland

43: Also at Gaziosmanpasa University, Tokat, Turkey

44: Also at Adiyaman University, Adiyaman, Turkey

45: Also at Izmir Institute of Technology, Izmir, Turkey

46: Also at The University of Iowa, Iowa City, USA

47: Also at Mersin University, Mersin, Turkey

48: Also at Ozyegin University, Istanbul, Turkey

49: Also at Kafkas University, Kars, Turkey

50: Also at Suleyman Demirel University, Isparta, Turkey

51: Also at Ege University, Izmir, Turkey

52: Also at Mimar Sinan University, Istanbul, Istanbul, Turkey

53: Also at Kahramanmaras Sütcü Imam University, Kahramanmaras, Turkey

54: Also at School of Physics and Astronomy, University of Southampton, Southampton, United Kingdom

55: Also at INFN Sezione di Perugia; Università di Perugia, Perugia, Italy

56: Also at Utah Valley University, Orem, USA

57: Now at University of Edinburgh, Scotland, Edinburgh, United Kingdom

58: Also at Institute for Nuclear Research, Moscow, Russia

59: Also at University of Belgrade, Faculty of Physics and Vinca Institute of Nuclear Sciences, Belgrade, Serbia

60: Also at Argonne National Laboratory, Argonne, USA

61: Also at Erzincan University, Erzincan, Turkey

62: Also at KFKI Research Institute for Particle and Nuclear Physics, Budapest, Hungary

63: Also at Kyungpook National University, Daegu, Korea 